1

2

3

\title{
Optimization of mica surface hydroxylation in water vapor plasma monitored by optical emission spec- troscopy
}

Patrick Rupper*, Martin Amberg, Dirk Hegemann, Manfred Heuberger*

Laboratory for Advanced Fibers, Empa, Swiss Federal Laboratories for Materials Science and Technology, Lerchenfeldstrasse 5, 9014 St.Gallen, Switzerland.

* Corresponding authors.

E-mail addresses: patrick.rupper@empa.ch (P. Rupper), manfred.heuberger@empa.ch (M. Heuberger)

Keywords:

Hydroxyl surface functionalization

Mica surface modification

Water vapor plasma

Chemical derivatization X-ray photoelectron spectroscopy (XPS)

Optical emission spectroscopy (OES)

This document is the accepted manuscript version of the following article: Rupper, P., Amberg, M., Hegemann, D., \& Heuberger, M. (2020). Optimization of mica surface hydroxylation in water vapor plasma monitored by optical emission spectroscopy. Applied Surface Science, 509, 145362 (12 pp.). https://doi.org/10.1016/ j.apsusc.2020.145362

This manuscript version is made available under the CC-BY-NC-ND 4.0 license http://creativecommons.org/licenses/by-nc-nd/4.0/ 


\section{Introduction}

Mica is a naturally occurring, non-swelling, hard and inert mineral and consists of aluminosilicate layers with intercalated layers of charge-compensating potassium ions. Muscovite mica, as used in this work, has the chemical formula $\mathrm{KAl}_{2}\left(\mathrm{AlSi}_{3}\right) \mathrm{O}_{10}(\mathrm{OH})_{2}$. Within each layer, the atoms are connected by strong covalent silicon-oxygen and aluminum-oxygen bonds, whereas the basal layers are only held together by electrostatic and weaker van der Waals forces [1]. The crystal preferentially fractures along these weak ionic layers and atomically smooth surfaces of large areas can be exposed in a straightforward manner. Therefore, mica is often used as a model substrate for surface force measurements requiring atomic smoothness [2,3]. This property makes mica also a preferred substrate for imaging adsorbed molecular assemblies and proteins using atomic force microscopy (AFM) [4,5]. In addition, mica as an ultra-smooth surface is used in template-stripping procedures to achieve a surface with only Angstrom size topographic features for metal [6] and polymer films [7]. Recently, flat mica was applied as gate dielectric layer in carbon nanotube field-effect transistors to improve performance [8]. In order to expand the range of surface chemistries of this smooth substrate, a common strategy is to use self-assembled monolayer (SAM) surface modifications. Covalent bonding is preferred over the instability inherent to electrostatically bound surfactant films $[9,10]$. Parker and coworkers $[9,11]$ have pioneered low-pressure water vapor plasma treatments to introduce reactive silanol ( $\mathrm{Si}-\mathrm{OH})$ groups. They served as anchoring points for further chemical functionalization, e.g. coupling via chloroor ethoxysilane chemistry to form stable hydrophobic monolayers [11-13]. Several research groups continued along this line and used a water vapor plasma activation procedure to functionalize mica surfaces with alkylsilanes [10,14], amino- and ethylene oxide groups [15], polystyrenes [16], polymer brushes [17] and proteins [18] as well as for patterning of mica [19]. Recent- 
ly, coupling via organophosphonic acids was applied after water vapor plasma activation to prepare polyelectrolyte brushes [20]. As an advantage, water vapor plasma treatment is a clean, dry and relatively fast method which does not significantly alter the bulk properties of the treated substrate [1]. Moreover, insignificant increase in mica surface roughness has been observed under optimal water vapor plasma conditions $[11,16,18,21]$.

Both pure water vapor $[9,11,15,22]$ as well as mixtures of argon gas and water vapor $[10,12,13,16,18]$ have been used in numerous variants of activation conditions. The optimization of the plasma process, i.e. the maximization of the hydroxyl surface density on mica, was always based on trial-and-error, where for each set of experimental plasma parameters a mica sample had to be processed and subsequently ex-situ analyzed. The surface density of Si-OH was quantified via different methods: (I) by measuring water contact angles after silanization with a hydrophobic fluorocarbon silane [11], with trimethylchlorosilane [3,16] or with hexamethyldisilazane [22], (II) by directly measuring contact angles using ethylene glycol exhibiting a high surface affinity with silanols [10], (III) by time-of-flight secondary ion mass spectrometry (ToF-SIMS) recording $\mathrm{SiOH}^{+} / \mathrm{Si}^{+}$ratios [10,23], (IV) by Fourier transform infrared spectroscopy (FTIR) [2426], and (V) by X-ray photoelectron spectroscopy (XPS) quantification with a marker (often fluorine, sometimes sulfur or nitrogen) after chemical derivatization with the marker-containing silane $[9,11,18]$. Whereas methods (I) to (IV) only give an estimate of surface hydroxylation density, XPS is more quantitative and allows the determination of absolute surface functional densities. Despite the amount of performed studies, no clear correlation between the plasma activation parameters (applied power, water flow rate, pressure, and duration of exposure) and the resulting hydroxyl surface density was elucidated, mostly due to the lack of systematic infor- 
mation/control of the plasma process. For the modification of polymeric substrates, water vapor plasma treatment has been extensively studied in the past by Fisher and coworkers [27-29] applying optical emission spectroscopy (OES), mass spectrometry and laser-induced fluorescence to characterize the gas-phase species. They showed that $\mathrm{OH}$ radicals are involved in the hydrophilic surface modification. Fumagalli et al. [30] also used OES for studying the role of hydroxyl molecules in a water vapor plasma process applied for biomolecules surface decontamination.

To quantify molecular surface density, XPS is a method of choice. We note that in view of XPS peak shape analysis, the hydroxyl group signal is convoluted with other oxygen components of mica and thus not directly quantifiable; namely, there is hardly a significant energy shift between silanol and silicon oxide photoelectrons, both for the Si $2 \mathrm{p}$ as well as O 1s peaks [23,25,31-33]. Using a special peak deconvolution algorithm with the consideration of instrumental peak broadening, a binding energy shift of about $0.6 \mathrm{eV}$ was inferred in the oxygen $\mathrm{O} 1 \mathrm{~s}$ signal between $\mathrm{Si}$ O-Si and Si-OH for glass surfaces [34]. Pantano and coworkers [35] pointed out that such spectral deconvolution of the $\mathrm{O} 1 \mathrm{~s}$ peak is very sensitive to various artifacts or broadening due to the spectrometer function; peak fitting with slightly different constraints resulted in significantly different $\mathrm{Si}-\mathrm{O}-\mathrm{Si}$ and $\mathrm{Si}-\mathrm{OH}$ fractions. A more practical strategy for quantification of surface hydroxyl groups is based on selective chemical derivatization; namely, silanol groups are tagged with a marker element that is distinctively detected by XPS. For one, the reagent trifluoroacetic anhydride, which is often used to determine hydroxyl groups in polymers via chemical derivatization [36], is inapplicable to silanols because the derivatization reaction does not proceed well on oxidized silicon surfaces [37,38]. More suitable reagents are thus chloro- or alkoxysilanes, which form silanols in presence of residual trace water (from atmospheric mois- 
ture) [38-41]. Via condensation reactions (i.e. under the release of water), covalent siloxane $\left(\mathrm{Si}_{\text {surface }}-\mathrm{O}-\mathrm{Si} i_{\text {silane }}\right)$ bonds are formed between the silanols and the hydroxyl groups present on the mica surface $[17,40,42]$. Very recently, Ahn et al. theoretically investigated the adsorption characteristics of silanes on silica and showed that the siloxane bond formation is a spontaneous reaction [43]. Due to their higher susceptibility toward moisture and hence increased reactivity, chlorosilanes are often a better choice compared to alkoxysilanes [18,44]. For a correct quantification of the hydroxyl surface density, the usage of monofunctional silanes is imperative, as they are the only ones capable of selectively reacting 1:1 with an $\mathrm{OH}$-activated mica surface [45]. Diand trifunctional silanes bear the possibility of multi dentate chemisorption or undesired intermolecular cross-linking, leading to an ill-defined vertical multilayering and polycondensate of organosilanes [17,40,42,44,46,47]. Compared to a solution-based derivatization, performing the derivatization reaction in the gas phase is applicable to a broader range of substrates [44]. Multifunctional silanes appear throughout the literature for chemical derivatization of glass and silicon oxide substrates and they were mostly used for the qualitative detection of silanol groups $[31,37,48]$. For absolute quantification, monofunctional silanes like cyanopropyldimethylchlorosilane (nitrogen marker atom) $[15,25]$ or (tridecafluoro-1,1,2,2tetrahydrooctyl)dimethylchlorosilane (fluorine marker atom) $[9,11,49]$ have been used.

This work uses a water vapor plasma to create reactive silanol groups at the mica surface. As a new element, we have used optical emission spectroscopy (OES) as an online tool, which allows better monitoring/control of the interaction between the water vapor plasma and mica. The physical background is that a significant correlation exists between the pressure-normalized OES hydroxyl intensity and the emerging surface density of hydroxyl groups. Monitoring the OES and 
pressure signals thus allows efficient in-situ optimization of the water vapor plasma conditions and bypasses laborious iterations between plasma treatments and surface analytics. For a quantitative calibration of this method, the corresponding silanol surface density was carefully determined by applying gas-phase monofunctional derivatization XPS using (tridecafluoro-1,1,2,2tetrahydrooctyl)dimethylchlorosilane.

\section{Experimental section}

\subsection{Water vapor plasma treatment of mica}

Ruby mica blocks (grade \# 2) were purchased from S\&J Trading Inc., USA. Sheets of ca. 1 x 1 $\mathrm{cm}^{2}$ of freshly cleaved mica surfaces (final thickness of about $0.25 \mathrm{~mm}$ ) were prepared inside a laminar airflow cabinet, preventing dust deposition on the freshly exposed mica surfaces. The mica samples were immediately processed and either used for XPS (without or with an additional chemical derivatization step), for AFM measurements, or, for contact angle measurements, or, directly transferred into the plasma reactor. The latter consists of a cylindrical, symmetric reactor using capacitive coupling and low-pressure discharge radiofrequency (RF) excitation (13.56 $\mathrm{MHz})$. The plasma reactor embodies two parallel round electrodes $(\varnothing 30 \mathrm{~cm})$ separated by a glass ring ( $5 \mathrm{~cm}$ in height). The upper electrode contains a gas showerhead, while the chamber is pumped via a grid in the lower electrode. The mica substrate is placed on the lower electrode that couples to the RF generator as previously described [50]. Fig. 1 schematically depicts the experimental setup consisting of the plasma reactor and the supply of water vapor. A CEM device (controlled evaporation and mixing model W-102A) from Bronkhorst, Netherland was used. The quantity of ultrapure $(18.2 \mathrm{M} \Omega \cdot \mathrm{cm})$ water was defined by a liquid flow meter (LFC CORIFLOW model M12-ABD-22-0-S). The water was carried by Argon gas that was controlled by a 
mass flow controller (MFC EL-FLOW model F-201CV-050-ABD-22-V) to a fixed flow rate of $50 \mathrm{sccm}$ (standard cubic centimeters per minute) and heated to $80{ }^{\circ} \mathrm{C}$ (temperature controller E8000, E-8507-A-1WBTU). The water/Argon mixture was transferred into the plasma chamber via a heated $\left(70{ }^{\circ} \mathrm{C}\right)$ stainless steel transfer line. Using the $\mathrm{LFC}$, the water flow rate was set in gram per hour (g/h), which can be converted to sccm by using the ideal gas law (10 g/h corresponds to a water vapor flow rate of $210 \mathrm{sccm}$ ). Plasma input power was varied systematically over a range from $30 \mathrm{~W}$ to $150 \mathrm{~W}$ and liquid water flow rate from $2.5 \mathrm{~g} / \mathrm{h}$ to $10 \mathrm{~g} / \mathrm{h}$. In addition, the pump capacity was adjusted to control the chamber pressure in a range from 0.05 mbar to 0.9 mbar. The standard procedure included a plasma treatment duration of 5 minutes. A subsequent $\sim 5$ minutes pumping cycle, with gas inlet valves closed, was utilized to prevent adsorption of water on plasma-activated sites that could reduce the amount of silanol groups [16]. The mica surfaces were then re-pressurized with nitrogen gas and removed from the plasma chamber for the optional directly successive chemical derivatization step. A protocol of immediate transfer (within only few minutes) to the derivatization setup as well as for XPS, AFM or contact angle measurements was followed in order to prevent significant loss of Si-OH due to a condensation reaction between neighboring silanol groups.

For comparison, mica was also hydroxylated using known wet-chemical processes [42,51-54]. Freshly cleaved mica samples were either boiled in deionized water at $95{ }^{\circ} \mathrm{C}$ for 30 minutes or immersed in a piranha solution for 30 minutes at room temperature, both followed by rinsing with ethanol. The piranha solution was freshly prepared and consisted of a mixture between concentrated sulfuric acid and hydrogen peroxide $\left(3: 1 \mathrm{H}_{2} \mathrm{SO}_{4}\right.$ to $\left.\mathrm{H}_{2} \mathrm{O}_{2} \mathrm{v} / \mathrm{v}\right)$. 


\subsection{X-ray photoelectron spectroscopy (XPS)}

XPS measurements were performed with a scanning XPS microprobe spectrometer (PHI

VersaProbe II, Physical Electronics, Minnesota, U.S.) using monochromatic Al Ka radiation

$(1486.6 \mathrm{eV})$. The operating pressure of the XPS analysis chamber was below $5 \times 10^{-7} \mathrm{~Pa}$ for all data presented here. Survey scan spectra, higher resolution narrow region spectra and angleresolved X-ray photoelectron spectra (ARXPS) were acquired. The former two spectra types were collected at a photoemission take-off angle, $\theta=45^{\circ}$. The ARXPS take-off angles were $15^{\circ}$, $30^{\circ}, 45^{\circ}, 60^{\circ}$ and $75^{\circ}$, with respect to the surface plane. During ARXPS measurements, the analyzer acceptance angle is ca. \pm 5 degrees, whereas in the normal lense mode for survey and region scans, it is about \pm 20 degrees. Survey scan spectra $(0-1100 \mathrm{eV})$ were acquired with an energy step of $0.8 \mathrm{eV}$, an acquisition time of $160-300 \mathrm{~ms}$ per data point and an analyzer pass energy of $187.85 \mathrm{eV}$. Detail spectra were acquired of C 1s and K $1 \mathrm{~s}(278 \mathrm{eV}$ to $300 \mathrm{eV}), \mathrm{O} 1 \mathrm{~s}(523 \mathrm{eV}$ to $543 \mathrm{eV}), \mathrm{Si} 2 \mathrm{p}(94 \mathrm{eV}$ to $114 \mathrm{eV}), \mathrm{Al} 2 \mathrm{p}(65 \mathrm{eV}$ to $85 \mathrm{eV})$ and $\mathrm{F} 1 \mathrm{~s}(678 \mathrm{eV}$ to $698 \mathrm{eV})$ using an energy step of $0.125 \mathrm{eV}$ and acquisition times of $1.3 \mathrm{~s}$ to $2.4 \mathrm{~s}$ per data point at an analyzer pass energy of $29.35 \mathrm{eV}$. To partially compensate the longer total measurement time for ARXPS due to the sequential recording at five different take-off angles, the step width and pass energy were slightly increased to $0.25 \mathrm{eV}$ and $58.70 \mathrm{eV}$, respectively. The energy resolution (FWHM, full width at half-maximum height) measured on the silver $\mathrm{Ag} 3 \mathrm{~d}_{5 / 2}$ photoemission line is $2.2 \mathrm{eV}$ (for a pass energy of $187.85 \mathrm{eV}$ ), $0.9 \mathrm{eV}$ (for a pass energy of $58.70 \mathrm{eV}$ ) and $0.7 \mathrm{eV}$ (for a pass energy of $29.35 \mathrm{eV}$ ). Total acquisition times were approximately 5 minutes for survey scans, 30 minutes per set of elemental scans and 80 minutes for a complete ARXPS measurement. 
The samples adhered to a stainless steel holder via double-sided adhesive tape. Randomly chosen measurement surface positions were analyzed using a micro-focused X-ray beam of diameter $100 \mu \mathrm{m}$ (operated at a power of $25 \mathrm{~W}$ at $15 \mathrm{kV}$ ). The $180^{\circ}$ spherical capacitor energy analyzer was operated in the fixed analyzer transmission (FAT) mode. Sample charging of the mica samples was compensated using dual beam charge neutralization with a flux of low energy electrons $(\sim 1 \mathrm{eV})$ combined with very low energy positive Ar ions $(10 \mathrm{eV})$. The binding energy is referenced to the C-C, $\mathrm{C}-\mathrm{H}$ hydrocarbon moiety signal (aliphatic $\mathrm{C}$ 1s peak at $285.0 \mathrm{eV}$ ). Intensity determination and curve fitting was carried out with CasaXPS software version 2.3.16 (Casa Software Ltd, Teignmouth, UK) using a fixed 70\% Gaussian - 30\% Lorentzian product function to fit the XPS spectra. Atomic concentrations were calculated from XPS peak areas after subtracting a Shirley type background. Thereby, tabulated PHI sensitivity factors [55] corrected for our system's transmission function and analyzer asymmetry parameter (correction due to a different angle between X-ray source and analyzer) have been used for quantification. Relative uncertainties in the measured concentration are estimated to be approximately $\pm 10 \%$ (it can be significantly more at small concentration levels, i.e. $\leq 0.5$ atomic $\%$ ); the error estimate includes uncertainties in the background determination from the energy window setting and transmission function correction. As an example, the detection limit of fluorine (part of the here used derivatizing reagent) in a matrix of light elements is estimated based on the procedure given by Shard [56] as ca. 0.3 atomic $\%$ under our experimental conditions.

\subsection{Chemical derivatization and quantification of the hydroxyl surface density}

The mica samples (untreated as well as with an additional water vapor plasma treatment) were derivatized (silanization) by exposure to silane vapors at reduced pressures. (Tridecafluoro- 
1,1,2,2-tetrahydrooctyl)dimethylchlorosilane, $97 \%$, CAS 102488-47-1, density $1.473 \mathrm{~g} / \mathrm{ml}$, boiling point $189^{\circ} \mathrm{C}$ with the formula $\mathrm{CF}_{3}\left(\mathrm{CF}_{2}\right)_{5}\left(\mathrm{CH}_{2}\right)_{2} \mathrm{Si}\left(\mathrm{CH}_{3}\right)_{2} \mathrm{Cl}$ has been used as derivatizing reagent. For comparison, also a smaller molecule has been applied, namely $(3,3,3-$ trifluoropropyl)dimethylchlorosilane, $97 \%$, CAS $1481-41-0$, density $1.11 \mathrm{~g} / \mathrm{ml}$, boiling point $118{ }^{\circ} \mathrm{C}$ with the formula $\mathrm{CF}_{3}\left(\mathrm{CH}_{2}\right)_{2} \mathrm{Si}\left(\mathrm{CH}_{3}\right)_{2} \mathrm{Cl}$. Both reagents are monofunctional and were obtained from abcr $\mathrm{GmbH}$, Germany and used without further purification. In addition, (tridecafluoro-1,1,2,2-tetrahydrooctyl)trichlorosilane, 97\% (Sigma-Aldrich, Switzerland), CAS 78560-45-9, density $1.3 \mathrm{~g} / \mathrm{ml}$, boiling point $192{ }^{\circ} \mathrm{C}$ with the formula $\mathrm{CF}_{3}\left(\mathrm{CF}_{2}\right)_{5}\left(\mathrm{CH}_{2}\right)_{2} \mathrm{SiCl}_{3}$ as an analogous tri-functional derivatizing reagent has been benchmarked. For derivatization, the mica substrates were placed in a glass flask $\left(250 \mathrm{~cm}^{3}\right)$ which was connected via a valve to a small reservoir container (volume ca. $10 \mathrm{~cm}^{3}$ ) comprising a few drops $(1 \mathrm{ml})$ of the liquid derivatizing reagent. The flask with the mica substrates was evacuated by a mechanical pump to facilitate vaporization of the silanization reagent. After reaching a pressure of $\leq 0.5 \mathrm{mbar}$, the pump was disconnected and the valve to the reservoir container opened for a specific derivatization time (5 minutes, 10 minutes, 30 minutes or 3 hours). After exposure, the reservoir container was again isolated from the flask with the mica substrates and the pumping procedure was repeated before the samples were taken out. Subsequently, the derivatized samples were thoroughly rinsed with ethanol to remove any potentially physisorbed derivatizing reagents, leaving only silanes covalently bonded to mica. After rinsing, the samples were dried under a stream of nitrogen gas.

Herder and coworkers [57] developed a suitable methodology to use XPS for the quantitative determination of surfactant densities on mica based on a complete exchange of surface potassium ions. The method uses the known three-dimensional chemical structure and properties of mica 
precisely determining the number of exchangeable potassium ions per surface area, which is utilized as an internal standard. Namely, the fraction of the potassium photoelectron signal corresponding to the exchangeable potassium ions (labelled $F_{\mathrm{R}}$ ) can directly be calculated [57]. Therein, the term $F_{\mathrm{R}}$ amounts to 0.314 under our experimental conditions (take-off angle $\theta$ of $45^{\circ}$ ); this implies that from the experimentally measured potassium concentration of 4.0 at.\% (see Table 1), a fraction 1.0 at.\% results from the top surface alone. After the water vapor plasma treatment, a potassium concentration of 3.2 at.\% was measured, meaning that the plasma removed about $80 \%$ of the surface potassium ions. Moreover, we have observed that rinsing with ethanol after the derivatization procedure causes additional potassium removal (presumably dependent on the purity of the used solvent). With this additional information, the formula developed by Herder et al. [57] for a $100 \%$ surface ion exchange can also be applied here to determine the surface hydroxyl densities after a water vapor plasma treatment of mica:

$$
\Gamma_{\mathrm{OH}}=\frac{\frac{[\mathrm{F}]}{13}\left(\frac{d}{\lambda \sin \theta}\right) \cdot 2.1}{[\mathrm{~K}] \cdot F_{\mathrm{R}} \cdot\left[\exp \left(\frac{d}{\lambda \sin \theta}\right)-1\right]}
$$

where $\Gamma_{\mathrm{OH}}$ is the hydroxyl number density (molecules $\left./ \mathrm{nm}^{2}\right),[\mathrm{F}]$ and $[\mathrm{K}]$ are the fluorine and potassium atomic concentrations, respectively, $d$ is the thickness of the derivatized layer, $\lambda$ is the inelastic mean free path (IMFP) in the layer and $\theta$ is the angle between the sample surface and the detector in the XPS system. The three last parameters together form the reduced thickness $d /(\lambda \sin \theta)$, which was determined from $[\mathrm{K}]=[\mathrm{K}]_{\mathrm{o}} \exp (-d / \lambda \sin \theta)$ by measuring the potassium atomic concentration before $\left([\mathrm{K}]_{\mathrm{o}}\right)$ and after $([\mathrm{K}])$ the derivatization procedure. The digit 13 in Eq. (1) represents the number of fluorine atoms specific to the derivatizing reagent. 


\subsection{Atomic force microscopy (AFM)}

In order to study the influence of the water vapor plasma treatment on the mica surface roughness, atomic force microscopy (AFM) measurements have been conducted in air with an easyScan 2 microscope (Nanosurf AG, Switzerland). Topographical images in contact mode (10 $\mathrm{nN}$ ) on a surface area of $2 \times 2 \mu \mathrm{m}^{2}$ were acquired using commercial silicon cantilevers (resonance frequency of $15 \mathrm{kHz}$, spring constant of $0.27 \mathrm{~N} / \mathrm{m}$ ). Data analysis was performed using the open-source software Gwyddion version 2.51. A two-dimensional levelling of the data was first carried out by mean plane subtraction, followed by aligning the rows using a third order polynomial method and setting the minimum data value to zero. The root-mean-square area roughness $\left(S_{q}\right)$ values were obtained for the entire area of the images.

\subsection{Contact angle measurements}

The contact angles were used to compare changes in the wettability of the mica surfaces after water vapor plasma treatment. Static contact angle measurements were performed with a DSA25 device (Krüss, Germany) by depositing drops $(2 \mu \mathrm{L})$ of water (Chromasolv, Sigma-Aldrich, Switzerland), ethylene glycol (Merck, Germany) or diiodomethane (Sigma-Aldrich, Switzerland). Reported values are an average over about 10 contact angle determinations at different places on the sample surface.

\subsection{Optical emission spectroscopy (OES)}

Optical emission spectra were obtained in a wavelength range from $261 \mathrm{~nm}$ to $825 \mathrm{~nm}$ (2047 pixel) for different values of plasma input power, water flow rate and chamber pressure. Thereby, the spectrometer Avaspec 3260, connected with a glass fiber (Avant FC-UV600-2SR), ob- 
served the optical emission of the water vapor plasma, as seen through the glass ring (borosilicate, high transmission above $250 \mathrm{~nm}$ ) that separated the plasma electrodes. A two seconds integration time was applied and the emission lines were analyzed and assigned with the software Spectrum Analyzer [58].

\section{Results and discussion}

First, the surface of the mica substrate is characterized using XPS, AFM and contact angle measurements; the surface is prepared pristine and at different stages of the process, i.e. untreated as well as after water vapor plasma treatment, then both before and after the chemical derivatization procedure. For these initial methodological investigations, we fixed a set of plasma parameters, being aware that these are not yet optimized. The XPS analysis showed that derivatization is appropriate for surface chemical quantification. The derivatization process was optimized based on a study of the kinetics of the silanization reaction using different previously described reagents, which indicated the necessity to use monofunctional silanes for a proper hydroxyl surface quantification. The main topic of the paper is then described in the following. In the second section, we assess and discuss the in-situ OES method as an online tool for the optimization of the water vapor plasma process, which will reveal a quantitative correlation between pressure-normalized OES hydroxyl intensities and silanol surface densities on mica. The XPS spectra obtained with the optimized water vapor plasma conditions are then given as a comparison to the initial ones. Finally, the number density of molecular hydroxyl groups on the mica surface after water vapor plasma treatment optimized with OES is quantified and compared to wet-chemical hydroxylation processes. 


\subsection{Surface characterization of mica after water vapor plasma treatment}

The surface of mica was characterized and compared for freshly cleaved samples before and after a water vapor plasma treatment. We have applied $50 \mathrm{~W}$ for the plasma input power, $10 \mathrm{~g} / \mathrm{h}$ for the water flow rate (i.e. $210 \mathrm{sccm}$ with $50 \mathrm{sccm} \mathrm{Ar}$ ) and $0.6 \mathrm{mbar}$ for the chamber pressure as a fixed set of parameters for the 5 minutes plasma duration. These starting conditions have been chosen to be midrange of various values found in the literature applying water vapor plasma to functionalize mica with hydroxyl groups $[10,12,13,18,22]$. Fig. 2a depicts XPS survey scans for freshly cleaved mica, for mica after water vapor plasma treatment, for mica after derivatization and for mica after water vapor plasma treatment and derivatization. The corresponding elemental concentrations derived from these XPS survey scans are listed in Table 1. The derivatization was performed in vapor of (tridecafluoro-1,1,2,2-tetrahydrooctyl)dimethylchlorosilane for 30 minutes duration and subsequent rinsing with ethanol. The samples were homogeneous and exhibited no position-dependent variation in the XPS elemental compositions. Assuming an inelastic mean free path of $\lambda=2.5 \mathrm{~nm}$ for potassium in mica [59], the information depth of XPS $(3 * \lambda * \sin \theta)$ at a take-off angle $\theta=45^{\circ}$ amounts to $5.3 \mathrm{~nm}$, which is deep enough for a representative elemental composition for mica with a crystal periodicity of $1 \mathrm{~nm}[10,57,60]$. The elements silicon (Si), oxygen (O), aluminum ( $\mathrm{Al}$ ) and potassium (K) expected for mica are clearly observed in Fig. 2a. In addition, a carbon (C) signal has been detected, which results from surface contamination. It has been shown that mica surface easily picks up carbonaceous contaminants from the ambient environment and that after an air exposure of only two minutes, no further significant increase in carbon concentration occurs $[61,62]$. The time needed to transfer the freshly cleaved mica sample to the XPS pumping system is exceeding this time window. Hence, under our experimental conditions, it is not possible to prevent the formation of this expected carbon contamination lay- 
er. The carbon concentration of 7.0 at.\% determined in this work (see Table 1) agrees with values found in the literature $[61,63]$ and is only slightly above the value of 5.2 at.\% observed for mica in-situ cleaved in the XPS chamber [61]. This adventitious carbon was confirmed to be located at the outer surface of mica because ARXPS at grazing incidence (take-off angle of $15^{\circ}$ ) led to an enhanced carbon to silicon ratio $(\mathrm{C} / \mathrm{Si})$ by a factor of $\sim 2.7 \mathrm{x}$ larger compared to the standard photoemission take-off angle of $45^{\circ}$, which closely matches the expected ratio $\sin \left(45^{\circ}\right) / \sin \left(15^{\circ}\right)=2.732$. Sodium $(\mathrm{Na})$, magnesium $(\mathrm{Mg})$ and iron $(\mathrm{Fe})$ as common impurities found in natural mica $[60,61]$ have also been detected in small concentrations $(\leq 0.2$ atomic $\%)$. The energetic positions of these elements, hardly visible at the chosen intensity scale, are marked with asterisks in Fig. 2a. The atomic concentrations of the freshly cleaved mica sample given in Table 1 are in good agreement with the theoretical values for ideal muscovite mica $\mathrm{KAl}_{2}\left(\mathrm{AlSi}_{3}\right) \mathrm{O}_{10}(\mathrm{OH})_{2}$ as well as with previously reported values $[63,64]$. Water vapor plasma treated mica showed a clear fluorine signal after chemical derivatization, whereas for derivatized mica without prior plasma treatment, only a very weak fluorine signal was observed ([F] $\leq 0.2$ at.\%, see Table 1); i.e. at the detection limit of XPS under our conditions. As expected, no fluorine has been detected for non-derivatized, freshly cleaved mica, both before and after water vapor plasma, confirming that it originates from the derivatizing reagent alone.

To quantify further the changes induced by plasma treatment and/or derivatization, higherresolution elemental scans were obtained as illustrated in Figs. $2 b-2 f$. The dotted lines represent the composite peaks, which were fitted according to the bond types expected for mica and its modification. Before derivatization, the element carbon is present from adventitious carbon contamination, therefore C-C/C-H $(285.0 \mathrm{eV}), \mathrm{C}-\mathrm{O}(286.4 \mathrm{eV})$ and $\mathrm{O}-\mathrm{C}=\mathrm{O}(289.1 \mathrm{eV})$ groups have 
been assigned (see Fig. $2 \mathrm{~b}$ ), and the component peak energy was restrained to within $\pm 0.3 \mathrm{eV}$ of corresponding literature values [65]. The major contribution to the spurious carbon signal resulted from aliphatic hydrocarbons (C-C, C-H) with minor parts of oxidized carbon C-O $(\leq 20 \%)$ and $\mathrm{O}-\mathrm{C}=\mathrm{O}(\leq 5 \%)$, in agreement with results from the literature $[1,61,63]$. The concentration and composition of carbon was comparable before and after water vapor plasma treatment, indicating that a very similar deposit of carbon contamination was formed during the transfer from the plasma reactor to the XPS chamber as on freshly cleaved mica. After derivatization, the water vapor plasma treated mica sample exhibited a significantly higher carbon signal, as expected due to the binding of the carbon-containing derivatizing reagent. In the case of non-optimized water vapor plasma conditions, most of the carbon signal still results from adventitious carbon on the mica substrate in-between the derivatized silanol groups. However, for optimized plasma parameters, nearly all of the carbon comes from the silanes itself due to the formation of a closed-packed monolayer of the derivatization molecules.

For the oxygen signal, the following bands are expected for untreated mica and for water vapor plasma treated mica; they were fitted in energy intervals according to literature values [34,60,64,66-68]: aluminum oxide and nonbridging $\mathrm{Si}^{-} \mathrm{O}^{-}$groups $(529.8-531.5 \mathrm{eV})$; aluminum hydroxyls, bridging Si-O-Si groups and O-C from adventitious carbon $(531.7-532.6 \mathrm{eV})$; $\mathrm{Si}-$ $\mathrm{OH}(0.6 \mathrm{eV}$ higher in energy than $\mathrm{Si}-\mathrm{O}-\mathrm{Si}$, as taken from Ref. [34]); water $(533.5-535.0 \mathrm{eV})$. Fig. 2c shows that the $\mathrm{O} 1 \mathrm{~s}$ peak is rather symmetrical with no clear separation into components, which is due to the partial overlap of above mentioned functional oxygen groups. This insufficient separation makes a rigorous quantitative analysis difficult, as already explained in the introduction. The $\mathrm{Si}$ signal is also not suitable to distinguish $\mathrm{Si}-\mathrm{O}-\mathrm{Si}$ from $\mathrm{Si}-\mathrm{OH}$, due to a small 
energy shift [32]; the convoluted signal has been fitted using literature values $[69,70]$ with two components as depicted in Fig. 2d, i.e. non-bridging $\mathrm{Si}^{-} \mathrm{O}^{-}(100.0-102.1 \mathrm{eV})$ and bridging Si-OSi plus Si-OH $(102.8-103.6 \mathrm{eV})$. Therefore, the $\mathrm{O}$ 1s and Si 2p data could be used in a qualitative way: namely, both the $\mathrm{O}$ 1s (Fig. 2c) and Si 2p (Fig. 2d) spectra exhibit a small energy shift of approximately $+0.5 \mathrm{eV}$ after water vapor plasma treatment (see vertical lines in Figs. $2 \mathrm{c}$ and 2d). This confirms the expected water-plasma-enhanced amount of silanols, which are located at higher binding energies, resulting in a shift of the overall peak maximum to higher energies. Along with this, a slight increase in the FWHM is observed for the $\mathrm{O}$ 1s and Si 2p peaks after water vapor plasma treatment. As expected, the spectrum for mica derivatized without a preceding water vapor plasma treatment is not significantly shifted relative to the reference mica. Quantitative characterization of silanols will be achieved using selective monofunctional chemical derivatization XPS (see later). The observed small increase of oxygen concentration (see Table 1) indicates successful incorporation of additional oxygen (presumably hydroxyl).

Aluminum (Fig. 2e) and potassium (Fig. 2b) spectra exhibited a symmetrical peak shape and were both freely fitted with only one bond type, corresponding to $\mathrm{Al}$ ions $(74.2 \mathrm{eV})$ and $\mathrm{K}$ ions (292.9 $\mathrm{eV}$ for the $\mathrm{K} 2 \mathrm{p}_{3 / 2}$ spin-orbit component) in mica. Table 1 shows that after water vapor plasma treatment, the amount of potassium and aluminum decreased relative to silicon, in agreement with results found by Parker and coworkers $[9,11]$. Because the outermost potassium layer is only held by ionic bonding and the covalent $\mathrm{Al}-\mathrm{O}$ bond in mica is weaker than the covalent $\mathrm{Si}-\mathrm{O}$ bond [61], both $\mathrm{K}$ and $\mathrm{Al}$ are preferential sputtered away from the mica surface in the plasma environment [64]. In addition, after the depletion of potassium, the resulting excess negative charge is compensated by a loss of surface aluminum or oxygen [9]. We note that the ob- 
served changes in chemistry only involve the outermost surface of mica, since the reduction of potassium signal corresponds to the amount of exchangeable surface potassium ions (see Eq. (1)). This is in agreement with observations made by Parker and coworkers using interferometric measurements [11]. As depicted in Figs. $2 \mathrm{~b}$ and 2e, the binding energies of $\mathrm{K}$ and $\mathrm{Al}$ increased slightly about $0.6 \mathrm{eV}$ and $0.3 \mathrm{eV}$, respectively (see vertical lines in Fig. 2) after water vapor plasma treatment, while the spectrum is nearly unchanged for the mica sample after derivatization in absence of prior plasma pretreatment. In case of the aluminum signal, we attribute this small binding energy shift to a slight change in the aluminum environment at the surface induced by the plasma process. Dehydration of Al-OH in mica, as reported after a water vapor plasma treatment [10], was not significant in this work (no peaks observable at higher binding energies of $76.5 \mathrm{eV}$ ). For potassium, the peak shift to higher binding energies after a water vapor plasma treatment coincides with a reduction in the FWHM of the peak from $\sim 1.8 \mathrm{eV}$ to $\sim 1.5 \mathrm{eV}$ due to the plasma removal of surface potassium ions as discussed before in Section 3.1. In addition, potassium ions close to the surface might either react with hydroxyl groups (being present in the water vapor plasma environment) or also with fluorine (produced by interaction of X-ray radiation with the derivatizing molecule) to form $\mathrm{KOH}$ and $\mathrm{KF}$, respectively, both species possessing a higher binding energy compared to surface potassium in mica due to the electronegativity of $\mathrm{OH}$ and $\mathrm{F}[71,72]$. The X-ray induced degradation of fluorine has been further investigated by consecutive survey scans at the same measurement spot. For a typical survey scan with a duration of 4 minutes, a decrease of $10 \%$ in the F 1s signal is observed (see Fig. S1 in the Supplementary material). The F 1s signal loss grows to $40 \%$ and $60 \%$ for 30 minutes and 80 minutes duration of X-ray exposure, respectively. It is known that fluorine has a high degradation index since its binding electrons have a significant cross-section for excitation by X-ray radiation [65]. 
The fluorine F 1s signal of the derivatizing reagent contains binding states around $688.8 \mathrm{eV}$ relating to $\mathrm{C}-\mathrm{F}$ bonds (see Fig. $2 \mathrm{f}$ ). In the carbon $\mathrm{C} 1 \mathrm{~s}$ spectrum, such $\mathrm{CF}_{2}$ and $\mathrm{CF}_{3}$ groups appear in a binding energy range of $291 \mathrm{eV}-293 \mathrm{eV}$ [55]; unfortunately, it is partially masked by a strong potassium signal. After selective silanization, the C-F band is quantitatively linked to the hydroxyl surface density on mica (see also Eq. (1)). We find that this C-F band intensity exhibits a correlation with the experimental parameters of the water vapor plasma treatment as well as with the choice of the derivatizing molecule. An additional weak peak $(\leq 10 \%$ of the total fluorine signal) was detected in the F 1s spectrum (see Fig. 2f); namely in the energy range between 684 $\mathrm{eV}$ and $686 \mathrm{eV}$; it can be assigned to the K-F bond [55] and is in agreement with the analysis of the potassium spectra. The relative K-F fluoride band intensity (compared to C-F) was not much dependent on the water vapor plasma conditions but increased with ongoing XPS measurement time to reach around 50\% of the total fluorine signal. This is in agreement with above postulated C-F cleavage during XPS measurements and subsequent reaction of fluorine with surface potassium ions. Indeed, ARXPS measurements have shown that the photoelectrons representing C-F bonds from the derivatizing molecule are emitted at the outermost surface, whereas the K-F fluoride signal is generated at the mica surface, i.e. below the derivatizing layer. In order to minimize degradation of the derivatizing reagent during XPS measurements, short survey scans have been used. Furthermore, the signal quantifications were corrected for the $10 \%$ decrease of fluorine during the 4 minutes duration of this scan, as quantified above.

Table 2 compares the contact angles for freshly cleaved mica surfaces before and after water vapor plasma treatment. Fluids with variable polarity contrast the change in wetting contact angle, 
namely water, ethylene glycol and diiodomethane. Our results are comparable to the ones found by Giasson and coworkers [10]. In particular, a significant decrease of the contact angle was found for ethylene glycol after water vapor plasma treatment. This reduction qualitatively indicates successful hydroxylation of mica and hence confirms the XPS investigations, because ethylene glycol with its two hydroxyl groups exhibits a high surface affinity toward silanol groups [10]. Diiodomethane is an apolar solvent and only partially wetted the mica surface, resulting in a higher contact angle (see Table 2), whereas water exhibits a low contact angle on untreated mica substrate without significant change after the water vapor plasma treatment.

As mentioned in the introduction, the surface forces apparatus technique requires a high degree of surface smoothness. For this application, it is important that the initially atomically smooth surface of mica does not gain roughness by the water vapor plasma treatment. For a verification, Fig. 3 depicts AFM images before (Fig. 3a) and after (Fig. 3b) treatment by a water vapor plasma. No significant change in the surface morphology and no significant increase in the rootmean-square area roughness $\left(S_{q}\right)$ values $(0.09 \mathrm{~nm}$ and $0.10 \mathrm{~nm}$ before and after water vapor plasma treatment, respectively) have been found. This finding applies to all used sets of plasma parameters; including those conditions optimized for high silanol surface densities. A smooth topography was also reported in the past when applying a water vapor plasma to mica $[16,18,21]$. This suggests that the observed chemical changes with XPS are essentially limited to the outermost mica layer (i.e. $<0.3 \mathrm{~nm}$ ) as already discussed before and also pointed out by Parker and coworkers [11]. In conclusion, the water-plasma hydroxylated samples can still be considered as atomically smooth substrates for surface forces measurements. 
In order to use chemical derivatization in XPS as a quantitative hydroxyl-detector, one has to examine the silanol derivatization reaction, its kinetics as well as the post-handling. After the reaction, no chlorine has been found in XPS survey spectra, meaning that the monofunctional chlorosilane derivatizing molecule completely reacted with the mica hydroxyl groups. This reaction proceeds either by directly forming $\mathrm{Si}-\mathrm{O}-\mathrm{Si}$ bonds under the release of $\mathrm{HCl}$, or by a preceding hydrolysis in the presence of residual water, followed by the formation of Si-O-Si bonds under the release of water. After the derivatization reaction, no change in the fluorine concentration has been observed as a function of sample storage in air for one day, in agreement with the observed stability of the siloxane and C-F bonds under environmental conditions [10]. Fig. 4 depicts the surface fluorine concentration as a function of the derivatization reaction time, for two related reagents. Both contain thirteen $\mathrm{F}$ atoms, one is a monochlorosilane (Fig. 4a), the other a trichlorosilane derivatizing reagent. The conversion curves are compared for untreated (open symbols and dotted lines) as well as water vapor plasma treated (filled symbols and permanent lines) mica substrates. The plasma parameters were $50 \mathrm{~W}$ input power, $10 \mathrm{~g} / \mathrm{h}$ water flow rate and 0.6 mbar chamber pressure. Fig. 4 a shows that the derivatization reaction was fast and a pronounced fluorine signal for the plasma treated sample was already observable after 5 minutes of derivatization. After ca. 30 minutes, the fluorine concentration reached its asymptotic value. Rinsing with ethanol led to a clear reduction in the fluorine concentration $(\approx 50 \%)$ for the plasma treated mica derivatized with the monochlorosilane reagent. This means that a significant portion of the derivatizing molecule was only physisorbed. Also for non-plasma activated mica, physisorbed derivatizing molecules were present (compare dotted lines in Fig. 4a) and could hence be rinsed off. A comparison with a smaller reagent containing the same reactive group (dimethylchlorosilane) but only three F atoms in the tail showed a very similar kinetics of the 
derivatization reaction with a fast fluorine increase followed by an asymptotic behavior. In agreement with the reduced number of $\mathrm{F}$ atoms, about four times less fluorine signal was detected.

Significant quantitative differences were observed between the monochloro- and trichlorosilane derivatizing reagents, where the latter one resulted in a seven times higher apparent fluorine atomic concentration (compare Figs. $4 \mathrm{a}$ and $4 \mathrm{~b}$ ). As expected, the monofunctional reagent formed a monolayer: ARXPS measurements, which we have performed, showed that carbon and fluorine (from the reagent) were mostly found at the outer surface. Oxygen, silicon, potassium and aluminum concentrations were lowest at grazing angle with subsequent increase and stabilization toward the deeper region, which mainly represents the bulk mica composition. However, as discussed in the introduction, for multifunctional chlorosilane derivatization, intermolecular crosslinking can occur $[18,46]$, which is seen as an excess signal of fluorine. Before rinsing, nearly the same high amount of fluorine is introduced by the derivatization, independent of the silanol-enriching water vapor plasma treatment (see Fig. 4b). Intermolecular crosslinking between multifunctional derivatizing molecules is thus more relevant than the covalent bonding of the reagent to silanol groups at the mica surface. Tripp and Hair confirmed on silica surfaces that less than $10 \%$ of the adsorbed trichloromethylsilane molecules were linked through a covalent $\mathrm{Si}_{\text {surface}}-\mathrm{O}-\mathrm{Si}$ bond, whereas about $70 \%$ of a monofunctional trimethylchlorosilane reacted with the surface silanol groups [40]. The tendency for intermolecular crosslinking was explained in terms of differing basicity of the $\mathrm{Si}-\mathrm{OH}$ groups in the two reagents as well as to a difference in kinetics between self-condensation and reaction with surface silanols. 
Altogether, our results confirm successful hydroxylation of a mica surface using a water vapor plasma treatment. For the remainder of this work, a standardized derivatization procedure with 30 minutes reaction time was used, followed by a rinsing step with ethanol. To this end, we shall establish OES measurements as a quantitative in-situ tool to control the water vapor plasma process, aiming at producing on-demand hydroxyl surface densities as well as finding its maximum.

\subsection{OES investigation of the water vapor plasma process and application to mica}

Optical emission spectroscopy (OES) is used to study the water vapor plasma process in-situ to understand the influences of plasma energy input, water flow rate and pressure as well as the role of surface processes for mica surface hydroxylation. The applied plasma input power, the input water flow-rate and the chamber pressure have been varied and the corresponding changes in the OES signal intensities of different species have been recorded. No mica substrate was present in the vacuum chamber for these initial investigations. Then, a selection of water vapor plasma parameters were applied for a fixed time of 5 minutes.

As an example, Fig. 5 depicts the OES spectrum collected from the water vapor plasma with 100 $\mathrm{W}$ input power, $5 \mathrm{~g} / \mathrm{h}$ water flow rate, $0.3 \mathrm{mbar}$ chamber pressure and $50 \mathrm{sccm}$ Ar. The observed emission lines are in good agreement with previous work from Fisher and coworkers [27] who studied gas phase reactive species at plasma-polymer surfaces using an inductively coupled water vapor plasma. Clear emission lines are observed from Ar at wavelengths between $400 \mathrm{~nm}$ and $820 \mathrm{~nm}$ [73]. In addition, the spectrum showed emissions from atomic hydrogen (Balmer series at $656 \mathrm{~nm}, 486 \mathrm{~nm}$ and $434 \mathrm{~nm}$ ) [74]. Excited $\mathrm{OH}^{*}$ lines were observed at $306.4 \mathrm{~nm}$ and 308.7 $\mathrm{nm}$ (see inset in Fig. 5), which correspond to the following electron-impact reaction in the plas- 
ma: $\mathrm{e}^{-}+\mathrm{H}_{2} \mathrm{O} \rightarrow \mathrm{OH}^{*}+\mathrm{H}^{*}+\mathrm{e}^{-}[27,28,75]$. No emission lines from $\mathrm{O}$ atoms were observed.

Many bands with low intensity were detected throughout the spectrum between $310 \mathrm{~nm}$ and 500 nm and mostly result from weaker Ar bands [73], from $\mathrm{H}_{2}$ [74] as well as from $\mathrm{CO}$ [27] emission lines, which is probably due to desorption from the chamber walls.

OES spectra like the one shown in Fig. 5 were used to correlate in-situ OH intensities to plasma parameters. The most intense $\mathrm{OH}$ band at $308.7 \mathrm{~nm}$ (see inset in Fig. 5) was selected for further analysis. Fig. 6 depicts this OES hydroxyl band intensity as an absolute value; a systematic variation of three water vapor plasma parameters is displayed in this data set. For the sake of clarity, only a representative selection of data is shown in Fig. 6, whereas Fig. S2 in the Supplementary material depicts the complete data set. The plasma duration was not varied, because no significant change has been observed in the hydroxyl surface density on mica by extending the plasma process duration beyond 5 minutes. The absolute OES hydroxyl intensity noticeably increased with plasma input power up to $100 \mathrm{~W}$ due to increased electron densities, while saturation can be observed for higher power values, probably due to gas heating. A power value of $100 \mathrm{~W}$ thus seems to be optimum for the formation of hydroxyl species in our plasma reactor. A lower water flow rate generally led to increased OES hydroxyl intensities. This relates to a longer residence time in the plasma before reaching saturation at higher power values of $100 \mathrm{~W}$ and $150 \mathrm{~W}$, and flow rates of $5 \mathrm{~g} / \mathrm{h}$ achieve similar or even slightly enhanced hydroxyl intensities compared to $2.5 \mathrm{~g} / \mathrm{h}$. Pressure variation, on the other hand, showed a minor effect on hydroxyl intensity in the plasma, probably due to an inherent balance between electron density and residence time. At 150 W input power, a lower pressure yielded lower hydroxyl intensity, according to a reduced residence time. No significant effect of the argon gas has been detected, as shown by the plotted ra- 
tio of OES signal intensities between $\mathrm{OH}$ and $\mathrm{Ar}$ (see $I_{-} \mathrm{OH} / I_{-} \mathrm{Ar}$ in Fig 6). For the argon intensity, the strong band at $750 \mathrm{~nm}$ was used for analysis (see Fig. 5).

According to Eq. (1), the measured fluorine concentration should be proportional to the hydroxyl surface density. It is thus interesting to compare the XPS fluorine signal with the OES hydroxyl intensities (Fig. 7a). The estimated relative uncertainties for the fluorine concentration are given as error bars and amount to $\pm 10 \%, \pm 20 \%$ and $\pm 40 \%$ for a fluorine content of $>2$ at. $\%, 0.6-2$ at. $\%$ and $\leq 0.5$ at. $\%$, respectively. A simple correlation is not readily visible; although an increase in the OES intensity of $\mathrm{OH}$ generally led to increased fluorine concentrations (see cone area marked in Fig. 7a). However, a surprisingly clear correlation appears, if we instead use the pressure-normalized OES hydroxyl intensity, as shown in Fig. 7b. A linear range of increased $[\mathrm{F}]$ (i.e. enhanced surface hydroxyl densities) vs. larger values of $I_{-} \mathrm{OH} / p$ is observed (see full line in Fig. $7 \mathrm{~b}$ ) up to a range where the fluorine atomic percentage has reached the monolayer limit. What plasma mechanism can explain such pressure dependence? According to literature data, $\mathrm{OH}$ species have a diffusion length (mean square displacement) of about $3 \mathrm{~cm}$ at a pressure of $\sim 2$ mbar, estimating a lifetime (half-life) of $\sim 10 \mathrm{~ms}$, whereby quenching by Ar is considered to be insignificant [76,77]. Therefore, the diffusion length is distinctly larger than the plasma-wall distance, i.e. the plasma sheath width, for our conditions and $\mathrm{OH}$ decay is thus only slightly depending on the used pressures (0.05- 0.9 mbar). Hence, surface processes for the mica surface hydroxylation need to be considered to explain the observed correlation of the fluorine signal with the pressure-normalized OES hydroxyl intensity. Most of all, the energy of incident ions (and fast neutrals) on the mica surface roughly scales with $p^{-1}$ in the considered pressure range due to collisions in the plasma sheath [78]. Increased ion bombardment at reduced pressure thus yield 
enhanced radical site formation, which enables the chemical binding of physisorbed radicals [79]. The here suggested dependence on radical site formation is underpinned by the finding that $\mathrm{OH}$ radicals per-se only show a moderate reactivity towards Si surfaces [80]. OHfunctionalization of mica in a water vapor plasma is thus a synergistic effect of $\mathrm{OH}$ radical density in the gas phase and highly energetic, non-equilibrium conditions at the surface.

Let us now discuss the quantification monolayer limit inherent to fluorine derivatization (see Fig. 7b). With the known orientation, methylsilane derivatizing molecules are expected to occupy a certain surface area that can be estimated by geometric arguments. At the limit of a close packed monolayer of the derivatization molecules, one has an equivalent of $2.5-3$ molecules $/ \mathrm{nm}^{2}$, as previously reported for the upper validity values for the $\mathrm{Si}-\mathrm{OH}$ surface density determinations applying methylsilane derivatization $[45,81]$. This methodological monolayer saturation limit is shown as dotted line in Fig. 7b. Its appearance is thus understood as an artefact of the chosen derivatization method; meaning that the true $-\mathrm{OH}$ surface densities can be higher. Higher surface hydroxyl densities are indeed reported for fully hydrated silica in the order of $4.5-5.5 \mathrm{OH} / \mathrm{nm}^{2}$; they are semi-quantitatively confirmed by other methods like secondary ion mass spectrometry, desorption studies, or considerations of the known crystallographic structure $[35,81])$. This upper $\mathrm{OH}$ limit cannot be detected via derivatization. We can expect that by further decreasing the plasma pressure, the final surface hydroxyl density will inevitably go through a maximum due to a decreased amount of particles ( $\mathrm{OH}$ radicals in the plasma). 


\subsection{Maximum (practical) hydroxyl surface density}

For the untreated mica substrate, the fluorine peak after derivatization was very weak (in the order of our detection limit of 0.2 at.\%, see Table 1), corresponding to a negligible silanol content of untreated mica. This is in agreement with observations that non-activated freshly cleaved mica surfaces remained unreactive toward silanization [11]. After applying a water vapor plasma treatment, a significant increase in the fluorine concentration after derivatization was found, which could be further optimized with the help of OES (see Fig. 7b). This is shown in Fig. 2, where XPS spectra for mica treated with initial and optimized water vapor plasma parameters are compared. A plasma input power of $100 \mathrm{~W}$, a water flow rate of $5 \mathrm{~g} / \mathrm{h}$ and chamber pressures of 0.2 mbar and lower yielded the highest detected fluorine concentrations ([F] $=12.0$ at. $\%$, see Table 1), which correspond to the methodological monolayer saturation limit (see before). An apparently saturating density of $2.7 \mathrm{Si}-\mathrm{OH} / \mathrm{nm}^{2}$ resulted using Eq. (1). Effective XPS-values for the potassium atomic concentration before and after the derivatization procedure are $[\mathrm{K}]_{\mathrm{o}}=3.2$ at. $\%$ and $[\mathrm{K}]=2.0$ at.\%, respectively; these values are used to derive the reduced thickness $d /(\lambda \sin \theta)$ in Eq. (1). Applying a derivatization procedure with methylchlorosilanes, the $\mathrm{OH}$ surface concentrations may thus be underestimated [45]. If two $\mathrm{Si}-\mathrm{OH}$ groups are at close proximity, the derivatization may effectively passivate the second group due to steric reasons. The probability for such occurrence increases with $\mathrm{OH}$ surface density and reaches values around unity at the monolayer saturation limit. Therefore, the $\mathrm{OH}$ surface density may continue to grow, while the fluorine signal no longer increases (see Fig. 7b). The maximum number density of passivated (undetected) $\mathrm{OH}$ groups can be estimated. Using the hexagonal arrangement of the mica surface Si-O top layer as given by Ostendorf et al. [62] and taking into account that 1 out of $4 \mathrm{Si}$ atoms is substituted by an $\mathrm{Al}$ atom [9], about $6 \mathrm{Si}$ atoms are present per $\mathrm{nm}^{2}$. This means that at the 
monolayer saturation limit, the density of $2.7 \mathrm{Si}-\mathrm{OH} / \mathrm{nm}^{2}$ includes at least $45 \%$ of all Si surface atoms that are potentially hydroxylated. Hence, the underestimation of the true hydroxyl surface density on mica due to a monolayer saturation limit from the derivatization is not more than about a factor of two.

We quantitatively compared the mica surface hydroxylation via water vapor plasma treatment with wet-chemical hydroxylation processes, i.e. boiling in water or immersing in a piranha solution. For the wet-chemical treatments, a reduced silicon content at the cost of a more pronounced adventitious carbon contamination was found (see Table S1 in the Supplementary material). Importantly, a ten times increased F/Si ratio has been obtained for the water vapor plasma treatment, making water-plasma the process of choice for mica surface hydroxylation. In the wetchemical treatment, the instability of silanol groups in water compared to their stability in air over some time $[10,11,43]$, may explain the unfavorably shifted equilibrium.

Finally, the hydroxyl surface density of $2.7 \mathrm{Si}-\mathrm{OH} / \mathrm{nm}^{2}$ achieved in this work can also be compared to literature values. Parker and coworkers $[9,11]$ determined a slightly lower value of 2.1 $\mathrm{Si}-\mathrm{OH} / \mathrm{nm}^{2}$ using the same derivatizing reagent. Their plasma parameters might have yielded a hydroxyl functionalization leading to a derivatization just below the monolayer saturation limit. Kiss and Gölander [15] applied a different monochlorosilane derivatizing reagent containing nitrogen instead of fluorine as marker atom and obtained a density of $1.0 \mathrm{Si}-\mathrm{OH} / \mathrm{nm}^{2}$ using Eq. (1). Okusa et al. [18] used a variety of multifunctional (bi- and trichloro as well as trialkoxysilanes) derivatizing reagents and obtained hydroxyl surface densities in the range of about 1 to $6 \mathrm{Si}$ $\mathrm{OH} / \mathrm{nm}^{2}$, which we would interpret as notorious overestimations due to cross-linking of the 
derivatizing reagent as discussed in Section 3.1. By optimizing the water vapor plasma process using in-situ OES measurements as performed in this work, the surface density of hydroxyl groups on mica reached the monolayer saturation limit for derivatization. Our results confirm that the maximum of the surface hydroxyl density achieved by water vapor plasma treatment on mica must be between 2.7 and $6 \mathrm{Si}-\mathrm{OH} / \mathrm{nm}^{2}$.

\section{Conclusions}

We successfully (re)used water vapor plasma treatments to systematically enrich the mica surface with hydroxyl functional groups and indirectly quantified the $-\mathrm{OH}$ surface density using monofunctional fluorinated derivatization in XPS. We found an upper detection limit for this method, which coincides with the monolayer saturation limit from derivatization. With the help of pressure-normalized optical emission spectroscopy (OES) signals, we could quantitatively control $\mathrm{OH}$ surface densities during plasma treatment. We thus identified both the $\mathrm{OH}$ density in the water vapor plasma as well as energetic plasma-surface interaction resulting in surface radical sites formation as key for achieving high hydroxyl surface densities. Applying an optimized water vapor plasma treatment, a hydroxyl surface density of $2.7 \mathrm{Si}-\mathrm{OH} / \mathrm{nm}^{2}$ on mica was reached. This value corresponds to the derivatization monolayer saturation limit; we anticipate that additional reduction of plasma pressure from 0.2 mbar down to $0.05 \mathrm{mbar}$ (at $100 \mathrm{~W}$ power input and $5 \mathrm{~g} / \mathrm{h}$ water flow rate) should further increase the $\mathrm{OH}$ surface density, beyond detection limit, towards the structural-stoichiometric maximum $\approx 6 \mathrm{Si}-\mathrm{OH} / \mathrm{nm}^{2}$. Because water vapor plasma treatment did not significantly change the surface roughness, mica with enhanced functional surface densities will enlarge its application as substrate for surface force measurements requiring atomically smooth surfaces. 


\section{Table 1}

Elemental composition $^{\mathrm{a}}$ for differently treated mica substrates obtained from XPS survey scans.

The values are given in atomic percentage concentrations ${ }^{\mathrm{b}}$ and have been normalized to $100 \%$.

\begin{tabular}{lcccccc}
\hline treatment of freshly cleaved mica & $\mathrm{C}$ & $\mathrm{O}$ & $\mathrm{Si}$ & $\mathrm{Al}$ & $\mathrm{K}$ & $\mathrm{F}$ \\
\hline none & $7.0(4)$ & $63.9(4)$ & $14.1(2)$ & $11.0(5)$ & $4.0(2)$ & - \\
water vapor plasma $^{\mathrm{c}}$ & $6.5(9)$ & $66.5(13)$ & $14.5(7)$ & $9.3(5)$ & $3.2(3)$ & - \\
derivatized $^{\mathrm{d}}$ & $7.1(14)$ & $63.5(8)$ & $14.3(6)$ & $11.2(7)$ & $3.8(4)$ & $0.1(1)$ \\
water vapor plasma and derivatized $^{\mathrm{c}, \mathrm{d}}$ & $10.3(1)$ & $62.9(8)$ & $13.1(2)$ & $9.6(7)$ & $2.6(4)$ & $1.5(3)$ \\
optimized plasma and derivatized $^{\mathrm{d}, \mathrm{e}}$ & $9.7(4)$ & $55.3(3)$ & $13.4(2)$ & $7.6(4)$ & $2.0(3)$ & $12.0(2)$ \\
\hline
\end{tabular}

${ }^{a}$ Sodium, magnesium and iron are only detected in minor concentrations $\leq 0.2$ at. $\%$ and have not been taken into account in the analysis.

$\mathrm{b}$ The errors are given in brackets and are one standard deviation in the average of three to five replicates.

${ }^{\mathrm{c}}$ Water vapor plasma treatment conditions were 5 minutes duration, $50 \mathrm{~W}$ input power, $10 \mathrm{~g} / \mathrm{h}$ water flow rate and 0.6 mbar pressure.

${ }^{\mathrm{d}}$ Derivatization using (tridecafluoro-1,1,2,2-tetrahydrooctyl)dimethylchlorosilane for 30 minutes duration and subsequent rinsing with ethanol.

${ }^{\mathrm{e}}$ Optimized water vapor plasma treatment conditions were 5 minutes duration, $100 \mathrm{~W}$ input power, $5 \mathrm{~g} / \mathrm{h}$ water flow rate and $0.2 \mathrm{mbar}$ pressure.

\section{Table 2}

Contact angles ${ }^{\mathrm{a}}$ of the three liquids water, ethylene glycol and diiodomethane measured for freshly cleaved and water vapor plasma treated mica substrates.

\begin{tabular}{lccc}
\hline $\begin{array}{l}\text { treatment of freshly } \\
\text { cleaved mica }\end{array}$ & water & ethylene glycol & diiodomethane \\
\hline none & $(5.5 \pm 3.7)^{\mathrm{O}}$ & $(25.5 \pm 4.9)^{\mathrm{O}}$ & $(34.4 \pm 4.0)^{\mathrm{o}}$ \\
water vapor plasma $^{\mathrm{b}}$ & $(8.3 \pm 3.5)^{\mathrm{O}}$ & $(11.8 \pm 1.5)^{\mathrm{O}}$ & $(31.1 \pm 5.3)^{\mathrm{o}}$
\end{tabular}

\footnotetext{
${ }^{a}$ The errors are one standard deviation in the average of 10 contact angle determinations at different areas on the mica sample surface.

${ }^{\mathrm{b}}$ Water vapor plasma treatment conditions were 5 minutes duration with $50 \mathrm{~W}$ input power, $10 \mathrm{~g} / \mathrm{h}$ water flow rate and 0.6 mbar pressure.
} 


\section{Declarations of interest}

None.

\section{Acknowledgements}

The authors would like to thank Thomas Niessen for his support with the measurement of the water contact angles.

\section{Appendix A. Supplementary material}

Supplementary data to this article can be found online.

\section{References}

[1] N.M.D. Brown, Z.-H. Liu, An investigation using atomic force microscopy and X-ray photoelectron spectroscopy of the modification of the surface of mica with an argon RF-plasma discharge, Appl. Surf. Sci. 90 (1995) 155-164.

[2] M. Heuberger, The extended surface forces apparatus. Part I. Fast spectral correlation interferometry, Rev. Sci. Instrum. 72 (2001) 1700-1707.

[3] M.W. Rutland, Surface forces between water-plasma-modified mica surfaces in solutions of non-ionic surfactant, Colloids Surf. A 83 (1994) 121-128.

[4] P.R. Heenan, T.T. Perkins, Imaging DNA equilibrated onto mica in liquid using biochemically relevant deposition conditions, ACS Nano 13 (2019) 4220-4229.

[5] A.S. Lea, A. Pungor, V. Hlady, J.D. Andrade, J.N. Herron, E.W. Voss, Manipulation of proteins on mica by atomic force microscopy, Langmuir 8 (1992) 68-73.

[6] N. Vogel, J. Zieleniecki, I. Köper, As flat as it gets: ultrasmooth surfaces from templatestripping procedures, Nanoscale 4 (2012) 3820-3832.

[7] D. Pires, B. Gotsmann, F. Porro, D. Wiesmann, U. Duerig, A. Knoll, Ultraflat templated polymer surfaces, Langmuir 25 (2009) 5141-5145.

[8] C.G. Low, Q. Zhang, Ultra-thin and flat mica as gate dielectric layers, Small 8 (2012) 21782183.

[9] J.L. Parker, D.L. Cho, P.M. Claesson, Plasma modification of mica: forces between fluorocarbon surfaces in water and a nonpolar liquid, J. Phys. Chem. 93 (1989) 6121-6125.

[10] B. Liberelle, X. Banquy, S. Giasson, Stability of silanols and grafted alkylsilane monolayers on plasma-activated mica surfaces, Langmuir 24 (2008) 3280-3288.

[11] J.L. Parker, P.M. Claesson, D.L. Cho, A. Ahlberg, J. Tidblad, E. Blomberg, Plasma modification of mica, J. Colloid Interface Sci. 134 (1990) 449-458. 

(1994) 2307-2310.

[13] S. Kim, H.K. Christenson, J.E. Curry, The effect of humidity on the stability of an octadecyltriethoxysilane monolayer self-assembled on untreated and plasma-treated mica, Langmuir 18 (2002) 2125-2129.

[14] T. Nakagawa, M. Soga, Contact angle and atomic force microscopy study of reactions of nalkyltrichlorosilanes with muscovite micas exposed to water vapor plasmas with various power densities, Jpn. J. Appl. Phys. 36 (1997) 6915-6921.

[15] E. Kiss, C.-G. Gölander, Chemical derivatization of muscovite mica surfaces, Colloids Surf. 49 (1990) 335-342.

[16] B. Liberelle, S. Giasson, Chemical end-grafting of homogeneous polystyrene monolayers on mica and silica surfaces, Langmuir 23 (2007) 9263-9270.

[17] B. Lego, W.G. Skene, S. Giasson, Unprecedented covalently attached ATRP initiator onto OH-functionalized mica surfaces, Langmuir 24 (2008) 379-382.

[18] H. Okusa, K. Kurihara, T. Kunitake, Chemical modification of molecularly smooth mica surface and protein attachment, Langmuir 10 (1994) 3577-3581.

[19] L. Dai, H.J. Griesser, A.W.H. Mau, Surface modification by plasma etching and plasma patterning, J. Phys. Chem. B 101 (1997) 9548-9554.

[20] O. Borozenko, V. Machado, W.G. Skene, S. Giasson, Organophosphonic acids as viable linkers for the covalent attachment of polyelectrolyte brushes on silica and mica surfaces, Polym. Chem. 5 (2014) 5740-5750.

[21] T.J. Senden, W.A. Ducker, Surface roughness of plasma-treated mica, Langmuir 8 (1992) 733-735.

[22] I.B. Malham, L. Bureau, Growth and stability of a self-assembled monolayer on plasmatreated mica, Langmuir 25 (2009) 5631-5636.

[23] B.J. Wood, R.N. Lamb, C.L. Raston, Static SIMS study of hydroxylation of low-surfacearea silica, Surf. Interface Anal. 23 (1995) 680-688.

[24] J. Peanasky, H.M. Schneider, S. Granick, C.R. Kessel, Self-assembled monolayers on mica for experiments utilizing the surface forces apparatus, Langmuir 11 (1995) 953-962.

[25] A.S. D'Souza, C.G. Pantano, K.M.R. Kallury, Determination of the surface silanol concentration of amorphous silica surfaces using static secondary ion mass spectroscopy, J. Vac. Sci. Technol. A 15 (1997) 526-531.

[26] A.S. D'Souza, C.G. Pantano, Hydroxylation and dehydroxylation behavior of silica glass fracture surfaces, J. Am. Ceram. Soc. 85 (2002) 1499-1504.

[27] M.L. Steen, C.I. Butoi, E.R. Fisher, Identification of gas-phase reactive species and chemical mechanisms occurring at plasma-polymer surface interfaces, Langmuir 17 (2001) 8156-8166.

[28] M.L. Steen, A.C. Jordan, E.R. Fisher, Hydrophilic modification of polymeric membranes by low temperature $\mathrm{H}_{2} \mathrm{O}$ plasma treatment, J. Membr. Sci. 204 (2002) 341-357.

[29] K.J. Trevino, J.C. Shearer, B.D. Tompkins, E.R. Fisher, Comparing isoelectric point and surface composition of plasma modified native and deposited $\mathrm{SiO}_{2}$ films using contact angle titrations and X-ray photoelectron spectroscopy, Plasma Process. Polym. 8 (2011) 951-964.

[30] F. Fumagalli, O. Kylián, L. Amato, J. Hanuš, F. Rossi, Low-pressure water vapour plasma treatment of surfaces for biomolecules decontamination, J. Phys. D: Appl. Phys. 45 (2012) 135203. 

of surface silanol in silicon nitride through chemical derivatization, J. Vac. Sci. Technol. A 9 (1991) 1406-1409.

[32] T. Gross, M. Ramm, H. Sonntag, W. Unger, H.M. Weijers, E.H. Adem, An XPS analysis of different $\mathrm{SiO}_{2}$ modifications employing a $\mathrm{C} 1 \mathrm{~s}$ as well as an $\mathrm{Au} 4 \mathrm{f}_{7 / 2}$ static charge reference, Surf. Interface Anal. 18 (1992) 59-64.

[33] E. Paparazzo, On the XPS analysis of Si-OH groups at the surface of silica, Surf. Interface Anal. 24 (1996) 729-730.

[34] D. Sprenger, H. Bach, W. Meisel, P. Gütlich, XPS study of leached glass surfaces, J. NonCryst. Solids 126 (1990) 111-129.

[35] J. Banerjee, S.H. Kim, C.G. Pantano, Elemental areal density calculation and oxygen speciation for flat glass surfaces using x-ray photoelectron spectroscopy, J. Non-Cryst. Solids 450 (2016) 185-193.

[36] P.-L. Girard-Lauriault, P. Dietrich, T. Gross, W.E.S. Unger, Is quantitative chemical derivatization XPS of plasma deposited organic coatings a valid analytical procedure?, Surf. Interface Anal. 44 (2012) 1135-1140.

[37] E.J. Szili, S. Kumar, R.S.C. Smart, R. Lowe, E. Saiz, N.H. Voelcker, Plasma enhanced chemical vapour deposition of silica onto titanium: Analysis of surface chemistry, morphology and hydroxylation, Surf. Sci. 602 (2008) 2402-2411.

[38] T.A. Dang, R. Gnanasekaran, Determination of amino and silanol functional groups on glass via chemical derivatization and ESCA, Surf. Interface Anal. 15 (1990) 113-118.

[39] J. Sagiv, Organized monolayers by adsorption. 1. Formation and structure of oleophobic mixed monolayers on solid surfaces, J. Am. Chem. Soc. 102 (1980) 92-98.

[40] C.P. Tripp, M.L. Hair, Reaction of methylsilanols with hydrated silica surfaces: the hydrolysis of trichloro-, dichloro-, and monochloromethylsilanes and the effects of curing, Langmuir 11 (1995) 149-155.

[41] M.F. Calhoun, J. Sanchez, D. Olaya, M.E. Gershenson, V. Podzorov, Electronic functionalization of the surface of organic semiconductors with self-assembled monolayers, Nature Mater. 7 (2008) 84-89.

[42] V. Dugas, Y. Chevalier, Surface hydroxylation and silane grafting on fumed and thermal silica, J. Colloid Interface Sci. 264 (2003) 354-361.

[43] Y.N. Ahn, S.H. Lee, S.Y. Oh, Adsorption characteristics of silane-functionalized perfluoropolyether on hydroxylated glassy silica surfaces: a multiscale approach, Appl. Surf. Sci. 496 (2019) 143699.

[44] J. Huang, X. Liu, X. Qiu, L. Xie, B. Yan, X. Wang, Q. Huang, H. Zeng, Octadecyltrichlorosilane deposition on mica surfaces: insights into the interface interaction mechanism, J. Phys. Chem. B 121 (2017) 3151-3161.

[45] P. Van Der Voort, S. Vercauteren, K. Peeters, E.F. Vansant, Some precautions when determining the silanol number, using chemical modification with methylchlorosilanes, J. Colloid Interface Sci. 157 (1993) 518-519.

[46] T.A. Dang, R. Gnanasekaran, D.D. Deppe, Quantification of surface hydroxides using chemical labeling and XPS, Surf. Interface Anal. 18 (1992) 141-146.

[47] R.G. Acres, A.V. Ellis, J. Alvino, C.E. Lenahan, D.A. Khodakov, G.F. Metha, G.G. Andersson, Molecular structure of 3-aminopropyltriethoxysilane layers formed on silanolterminated silicon surfaces, J. Phys. Chem. C 116 (2012) 6289-6297. 
[48] S. Takeda, M. Fukawa, Surface $\mathrm{OH}$ groups governing surface chemical properties of $\mathrm{SiO}_{2}$ thin films deposited by RF magnetron sputtering, Thin Solid Films 444 (2003) 153-157.

[49] N. Takahashi, H. Mori, Y. Kimoto, T. Ohmori, A. Murase, Analysis of silanol on DLC-Si surfaces by derivatization XPS, J. Surf. Sci. Soc. Jpn. 26 (2005) 492-494.

[50] D. Hegemann, M. Michlíček, N.E. Blanchard, U. Schütz, D. Lohmann, M. Vandenbossche, L. Zajíčková, M. Drábik, Deposition of functional plasma polymers influenced by reactor geometry in capacitively coupled discharges, Plasma Process. Polym. 13 (2016) 279-286.

[51] Y. Duvault-Herrera, N. Jaffrezic-Renault, P. Clechet, J. Serpinet, D. Morel, Quantitative study of the hydroxylation and of the chemical grafting of oxidized porous silicon, Colloids Surf. 50 (1990) 197-206.

[52] B. Bhushan, K.J. Kwak, S. Gupta, S.C. Lee, Nanoscale adhesion, friction and wear studies of biomolecules on silane polymer-coated silica and alumina-based surfaces, J. R. Soc. Interface 6 (2009) 719-733.

[53] C. Kinnear, J. Cadusch, H. Zhang, J. Lu, T.D. James, A. Roberts, P. Mulvaney, Directed chemical assembly of single and clustered nanoparticles with silanized templates, Langmuir 34 (2018) 7355-7363.

[54] L.L. Wang, Y.L. Liu, G.S. Liu, Hydrophobic coating of mica by piranha solution activation, silanization grafting, and copolymerization with acrylate monomers, J. Appl. Polym. Sci. 134 (2017) 44985.

[55] J.F. Moulder, W.F. Stickle, P.E. Sobol, K.D. Bomben, Handbook of X-ray Photoelectron Spectroscopy, Physical Electronics, Inc., Eden Prairie, MN, USA, 1995.

[56] A.G. Shard, Detection limits in XPS for more than 6000 binary systems using Al and Mg K $\alpha$ X-rays, Surf. Interface Anal. 46 (2014) 175-185.

[57] P.C. Herder, P.M. Claesson, C.E. Herder, Adsorption of cationic surfactants on muscovite mica as quantified by means of ESCA, J. Colloid Interface Sci. 119 (1987) 155-167.

[58] Z. Navrátil, D. Trunec, R. Šmíd, L. Lazar, A software for optical emission spectroscopy problem formulation and application to plasma diagnostics, Czech. J. Phys. 56 (2006) B944B951.

[59] S. Tanuma, C.J. Powell, D.R. Penn, Calculations of electron inelastic mean free paths. 3. Data for 15 inorganic compounds over the 50-2000 eV range, Surf. Interface Anal. 17 (1991) 927-939.

[60] K.G. Bhattacharyya, XPS study of mica surfaces, J. Electron Spectrosc. Relat. Phenom. 63 (1993) 289-306.

[61] Z.H. Liu, N.M.D. Brown, XPS characterization of mica surfaces processed using a radiofrequency (rf) argon plasma, J. Phys. D: Appl. Phys. 31 (1998) 1771-1781.

[62] F. Ostendorf, C. Schmitz, S. Hirth, A. Kühnle, J.J. Kolodziej, M. Reichling, How flat is an air-cleaved mica surface?, Nanotechnology 19 (2008) 305705.

[63] A. Keller, M. Fritzsche, R. Ogaki, I. Bald, S. Facsko, M.D. Dong, P. Kingshott, F. Besenbacher, Tuning the hydrophobicity of mica surfaces by hyperthermal Ar ion irradiation, J. Chem. Phys. 134 (2011) 104705.

[64] D. Bhowmik, P. Karmakar, Tailoring and investigation of surface chemical nature of virgin and ion beam modified muscovite mica, Surf. Interface Anal. 51 (2019) 667-673.

[65] G. Beamson, D. Briggs, High Resolution XPS of Organic Polymers, John Wiley \& Sons, Chichester, U.K., 1992.

[66] Z.-H. Liu, N.M.D. Brown, A. McKinley, Characterisation of oxygen plasma-modified mica surfaces using XPS and AFM, Appl. Surf. Sci. 108 (1997) 319-332. 
[67] A. Kowalewska, M. Nowacka, A. Tracz, T. Makowski, Supramolecular self-assembly of linear oligosilsesquioxanes on mica - AFM surface imaging and hydrophilicity studies, Soft Matter 11 (2015) 4818-4829.

[68] E. McCafferty, J.P. Wightman, Determination of the concentration of surface hydroxyl groups on metal oxide films by a quantitative XPS method, Surf. Interface Anal. 26 (1998) 549564.

[69] M. Cerruti, C.L. Bianchi, F. Bonino, A. Damin, A. Perardi, C. Morterra, Surface modifications of bioglass immersed in TRIS-buffered solution. A multitechnical spectroscopic study, J. Phys. Chem. B 109 (2005) 14496-14505.

[70] J. Abenojar, I. Colera, M.A. Martínez, F. Velasco, Study by XPS of an atmospheric plasmatorch treated glass: influence on adhesion, J. Adhes. Sci. Technol. 24 (2010) 1841-1854.

[71] M. Peuckert, On the adsorption of oxygen and potassium hydroxide on silver, Surf. Sci. 146 (1984) 329-340.

[72] W.E. Morgan, J.R. Van Wazer, W.J. Stec, Inner-orbital photoelectron spectroscopy of alkali metal halides, perchlorates, phosphates, and pyrophosphates, J. Am. Chem. Soc. 95 (1973) 751755.

[73] D.L. Crintea, U. Czarnetzki, S. Iordanova, I. Koleva, D. Luggenhölscher, Plasma diagnostics by optical emission spectroscopy on argon and comparison with Thomson scattering, J. Phys. D: Appl. Phys. 42 (2009) 045208.

[74] A. Granier, M. Vervloet, K. Aumaille, C. Vallée, Optical emission spectra of TEOS and HMDSO derived plasmas used for thin film deposition, Plasma Sources Sci. Technol. 12 (2003) 89-96.

[75] M.L. Steen, L. Hymas, E.D. Havey, N.E. Capps, D.G. Castner, E.R. Fisher, Low temperature plasma treatment of asymmetric polysulfone membranes for permanent hydrophilic surface modification, J. Membr. Sci. 188 (2001) 97-114.

[76] N.G. Adams, C.R. Herd, D. Smith, Development of the flowing afterglow/Langmuir probe technique for studying the neutral products of dissociative recombination using spectroscopic techniques: $\mathrm{OH}$ production in the $\mathrm{HCO}_{2}{ }^{+}+$e reaction, J. Chem. Phys. 91 (1989) 963-973.

[77] I. Yagi, R. Ono, T. Oda, K. Takaki, Two-dimensional LIF measurements of humidity and $\mathrm{OH}$ density resulting from evaporated water from a wet surface in plasma for medical use, Plasma Sources Sci. Technol. 24 (2015) 015002.

[78] A. Manenschijn, W.J. Goedheer, Angular ion and neutral energy distribution in a collisional rf sheath, J. Appl. Phys. 69 (1991) 2923-2930.

[79] H. Deutsch, H. Kersten, S. Klagge, A. Rutscher, On the temperature dependence of plasma polymerization, Contrib. Plasma Phys. 28 (1988) 149-155.

[80] P.R. McCurdy, K.H.A. Bogart, N.F. Dalleska, E.R. Fisher, A modified molecular beam instrument for the imaging of radicals interacting with surfaces during plasma processing, Rev. Sci. Instrum. 68 (1997) 1684-1693.

[81] D.W. Sindorf, G.E. Maciel, Cross-polarization magic-angle-spinning silicon-29 nuclear magnetic resonance study of silica gel using trimethylsilane bonding as a probe of surface geometry and reactivity, J. Phys. Chem. 86 (1982) 5208-5219. 


\section{Figure captions}

Figure 1. Schematic view of the experimental setup for the mica surface hydroxylation using a water vapor plasma treatment. It consists of the plasma reactor (containing the mica samples) and the CEM (controlled evaporation and mixing) device supplying the water vapor. LFC: liquid flow meter, $\mathrm{MFC}$ : mass flow controller, $\mathrm{LN}_{2}$ : liquid nitrogen.

Figure 2. Comparison of XPS spectra of freshly cleaved mica for different treatments. 1: none, 2: water vapor plasma, 3: derivatized, 4: water vapor plasma and derivatized, 5: optimized conditions for water vapor plasma and derivatized. The spectra are offset in the $y$-direction for better visualization. (a) Survey scans with a normalized O 1s signal intensity of one. Peak positions of sodium, magnesium and iron are marked with asterisks. (b) High-resolution scans (full lines) and deconvolution (dotted lines) for $\mathrm{C} 1$ s and $\mathrm{K} 2 \mathrm{p}$, (c) for $\mathrm{O} 1 \mathrm{~s}$, (d) for Si 2p, (e) for Al 2p and (f) for $\mathrm{F} 1 \mathrm{~s}$. Thin vertical lines mark the shift in binding energy due to the changed surface chemistry after water vapor plasma treatment (see text for details).

Figure 3. AFM images of mica (a) before and (b) after five minutes water vapor plasma treatment $(100 \mathrm{~W}$ input power, $5 \mathrm{~g} / \mathrm{h}$ water flow rate and $0.3 \mathrm{mbar}$ chamber pressure). The rootmean-square area roughness $\left(S_{q}\right)$ for the entire area $\left(2 \times 2 \mu \mathrm{m}^{2}\right)$ is given in the bottom part of the figure.

Figure 4. Derivatization conversion curves for untreated (open symbols) compared to water vapor plasma treated mica (filled symbols). The fluorine content as a function of the derivatization duration is given without and with an additional ethanol-rinsing step after derivatization. The points are connected by lines, which only serve as a guide for the eye. The estimated relative uncertainties for the measured fluorine concentration are given as error bars and amount to $\pm 5-10 \%, \pm 20 \%$ and $\pm 40 \%$ for a fluorine content of $>2$ at. $\%, 0.6-2$ at. $\%$ and $\leq 0.5$ at. $\%$, respectively. Derivatizing reagent used were (a) (tridecafluoro-1,1,2,2-tetrahydrooctyl)dimethylchlorosilane and (b) (tridecafluoro-1,1,2,2-tetrahydrooctyl)trichlorosilane.

Figure 5. OES spectrum of an $\operatorname{Ar}(50 \mathrm{sccm}) /$ water vapor $(5 \mathrm{~g} / \mathrm{h})$ plasma with $100 \mathrm{~W}$ input power. The chamber pressure was fixed at 0.3 mbar. The peak labels represent the assignments of the bands. The inset shows a zoom of the $\mathrm{OH}$ band around $310 \mathrm{~nm}$.

Figure 6. OES hydroxyl intensity as a function of a selection of various water vapor plasma conditions (input power, water flow rate, and chamber pressure). In addition, the OES intensity ratio between hydroxyl and argon is given on the right $y$-axis. All lines only serve as a guide for the eye.

Figure 7. Correlation diagrams between the XPS fluorine concentration after derivatization and, (a) the OES hydroxyl intensity, (b) the pressure-normalized OES hydroxyl intensity (i.e. intensity divided by the chamber pressure). The cone area in a) marks the general increase of [F] with $\mathrm{OH}$ intensity. A linear regression curve is displayed in b) for the first points up to the monolayer saturation limit (dashed line). The plasma chamber pressure (in mbar) is given at the measurement points and the legend depicts (color-coded) the other plasma parameters, i.e. input power and water flow rate. Open symbols concern data displayed in Fig. 6, whereas full symbols represent additional measurement points. 
Click here to download Figure: Figure1n.pptx

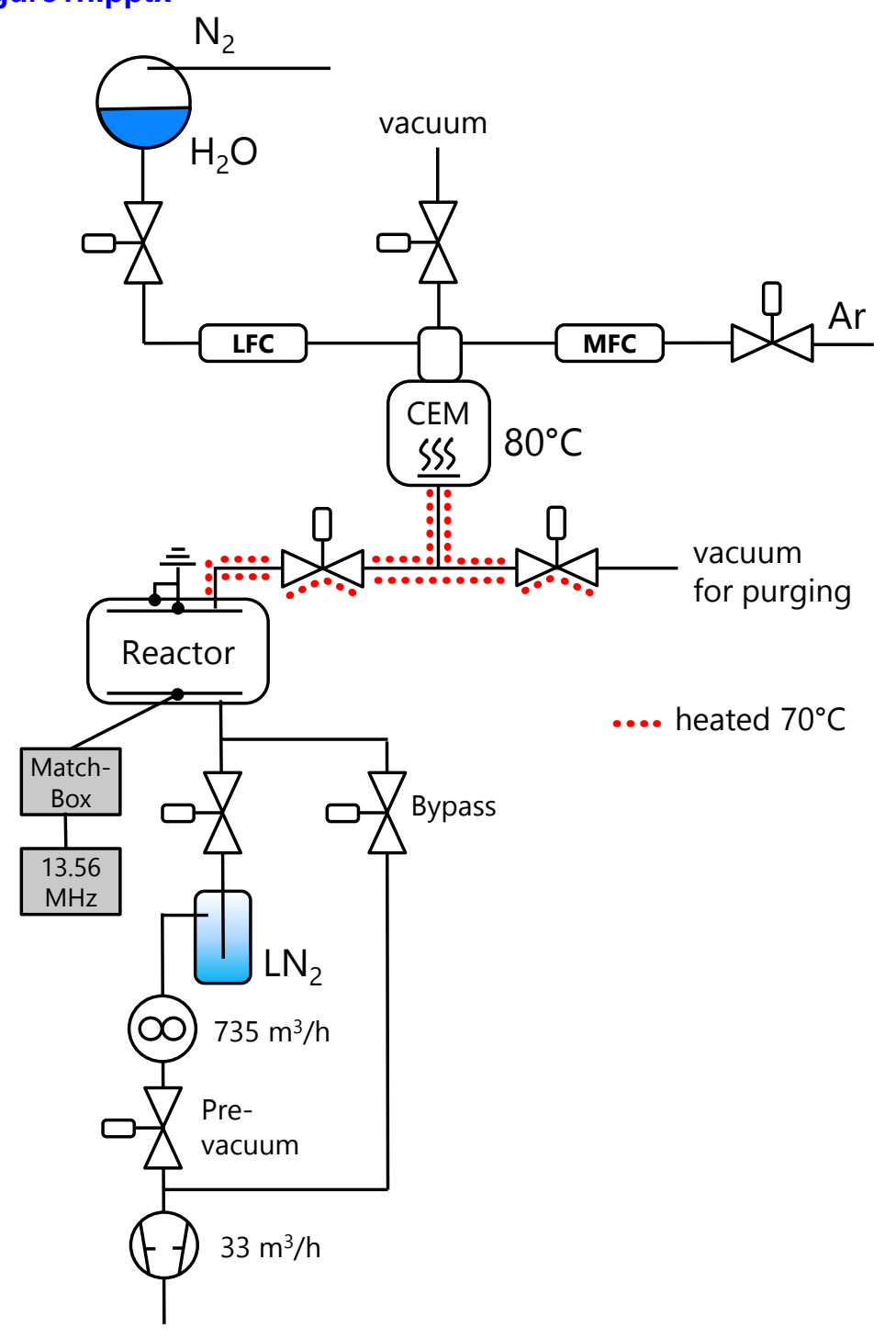

Figure 1 


\section{Figure 2}

Click here to download Figure: Figure2n_revised.pptx

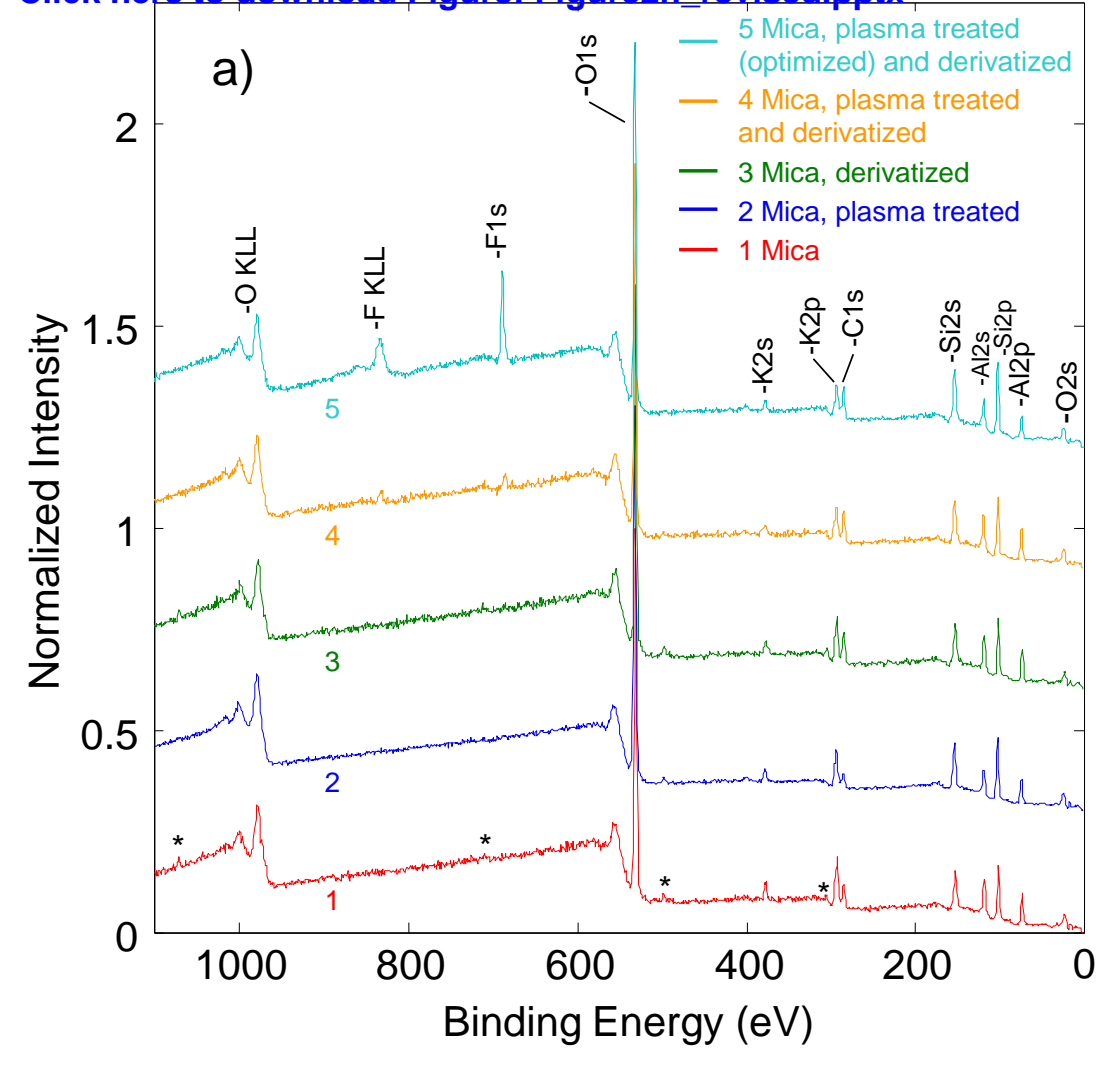

Figure 2
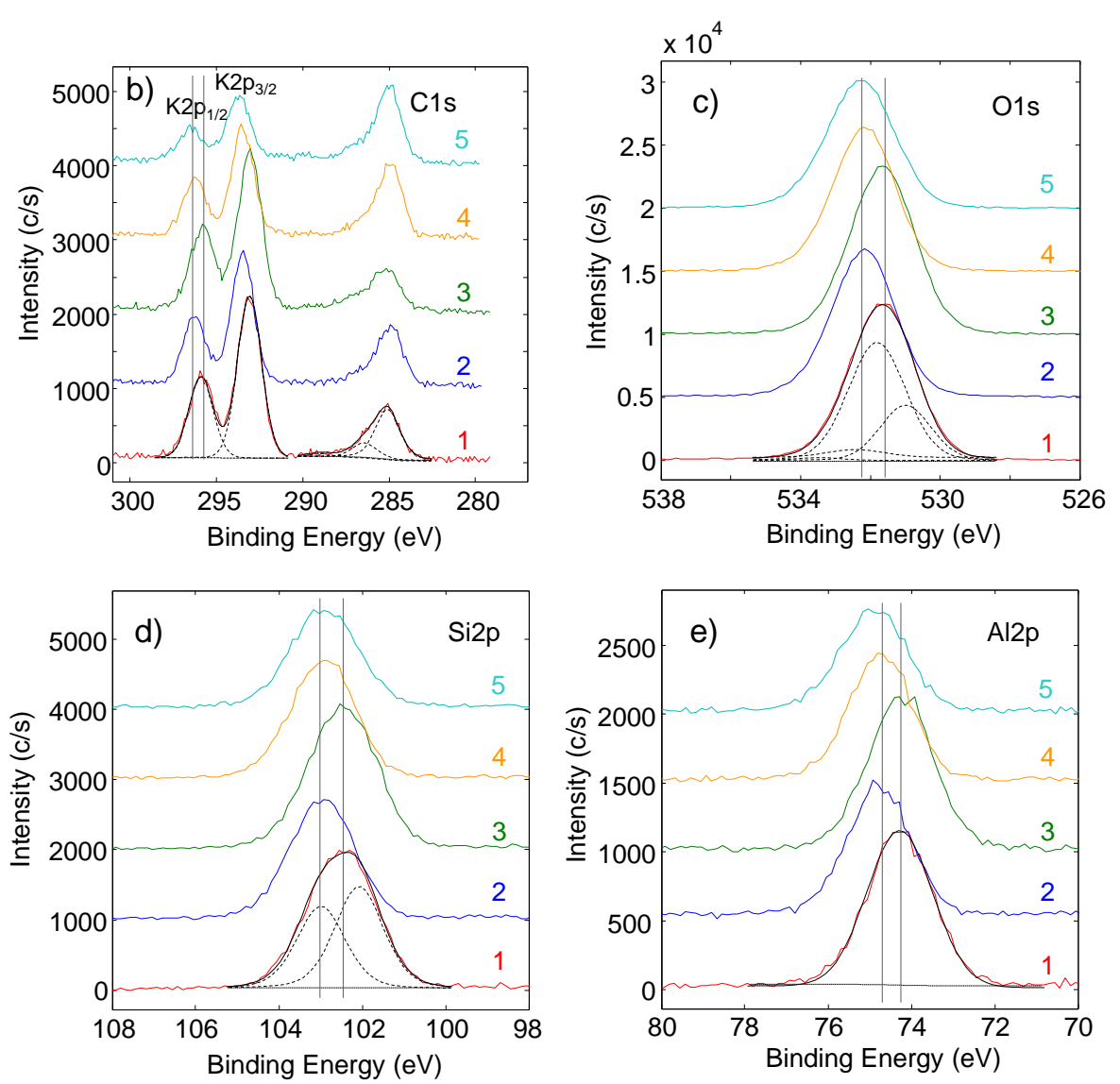

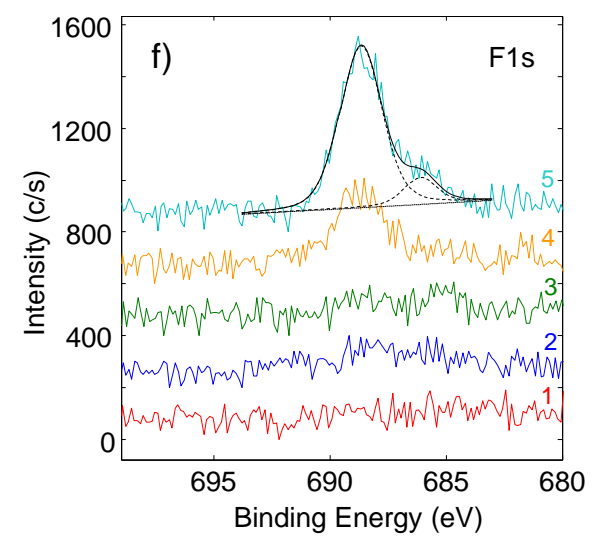


Figure

Click here to download Figure: Figure3n.pptx

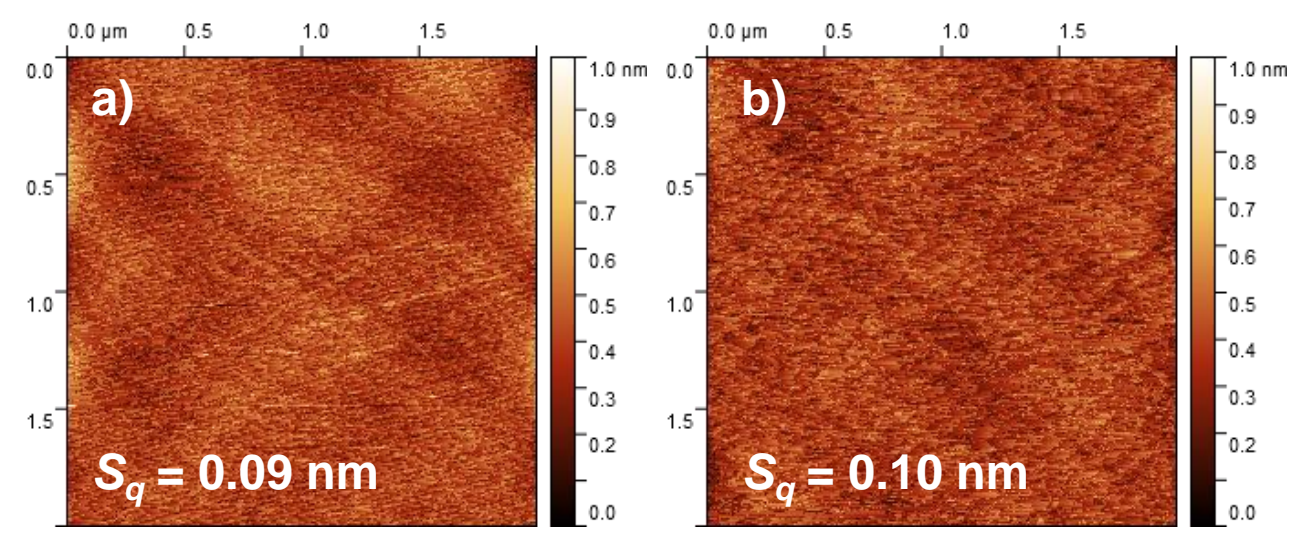

Figure 3 
Figure 4

Click here to фlownload Figure: Filgurure4pip.pptx
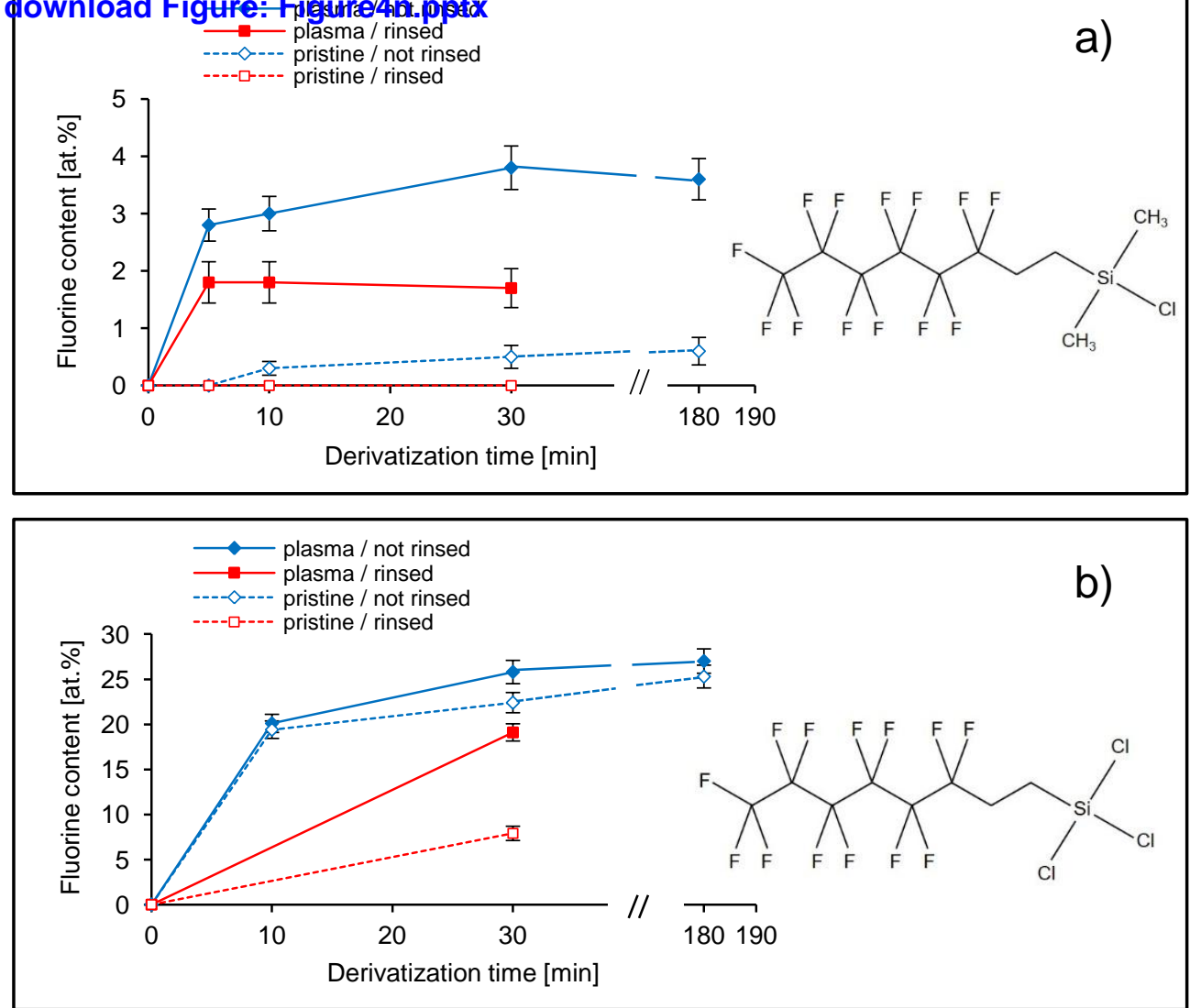

Figure 4 
Click here to download Figure: Figure5n.pptx

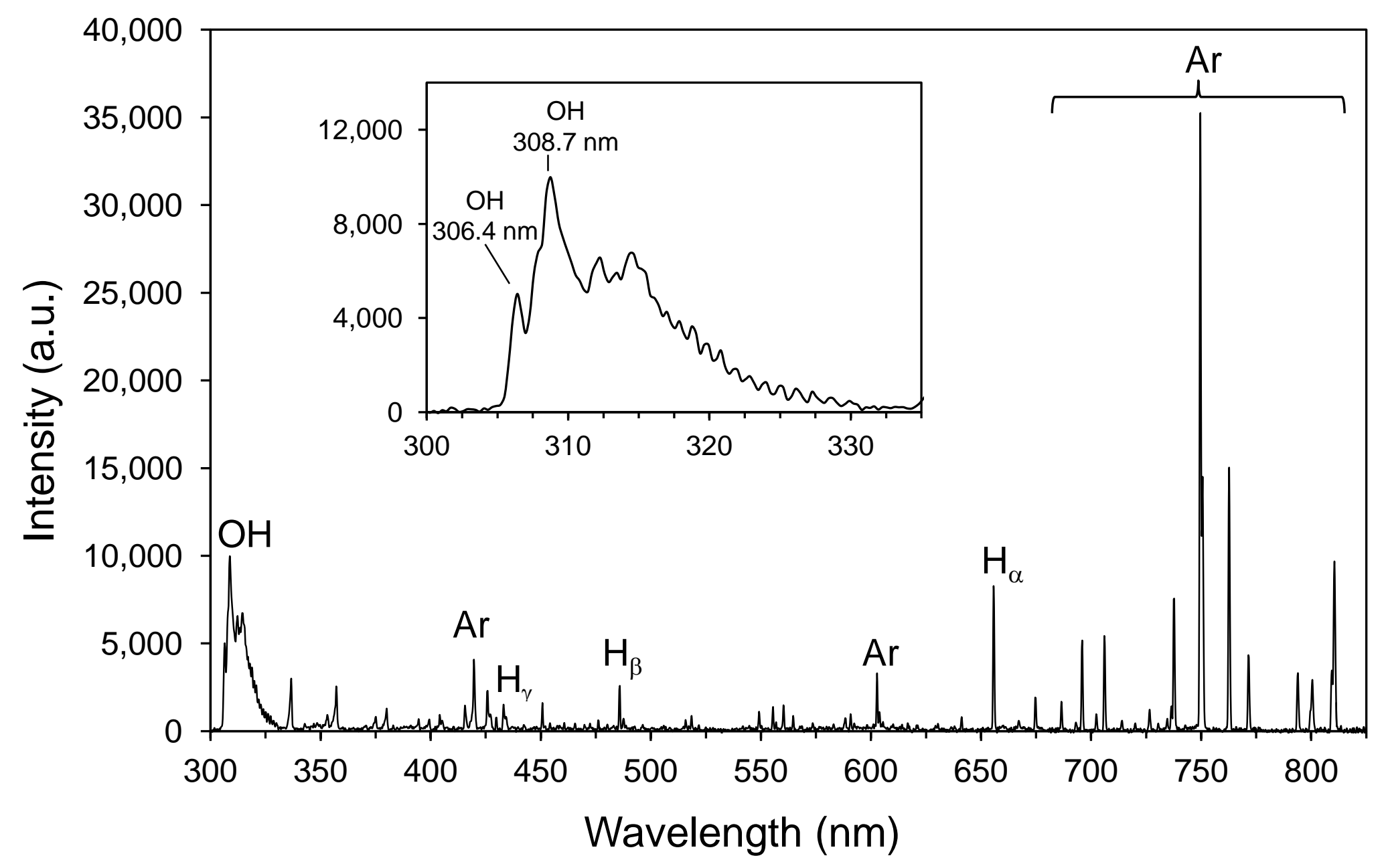

Figure 5 


\section{igure 6}

Click here to download Figure: Figure6n.pptx

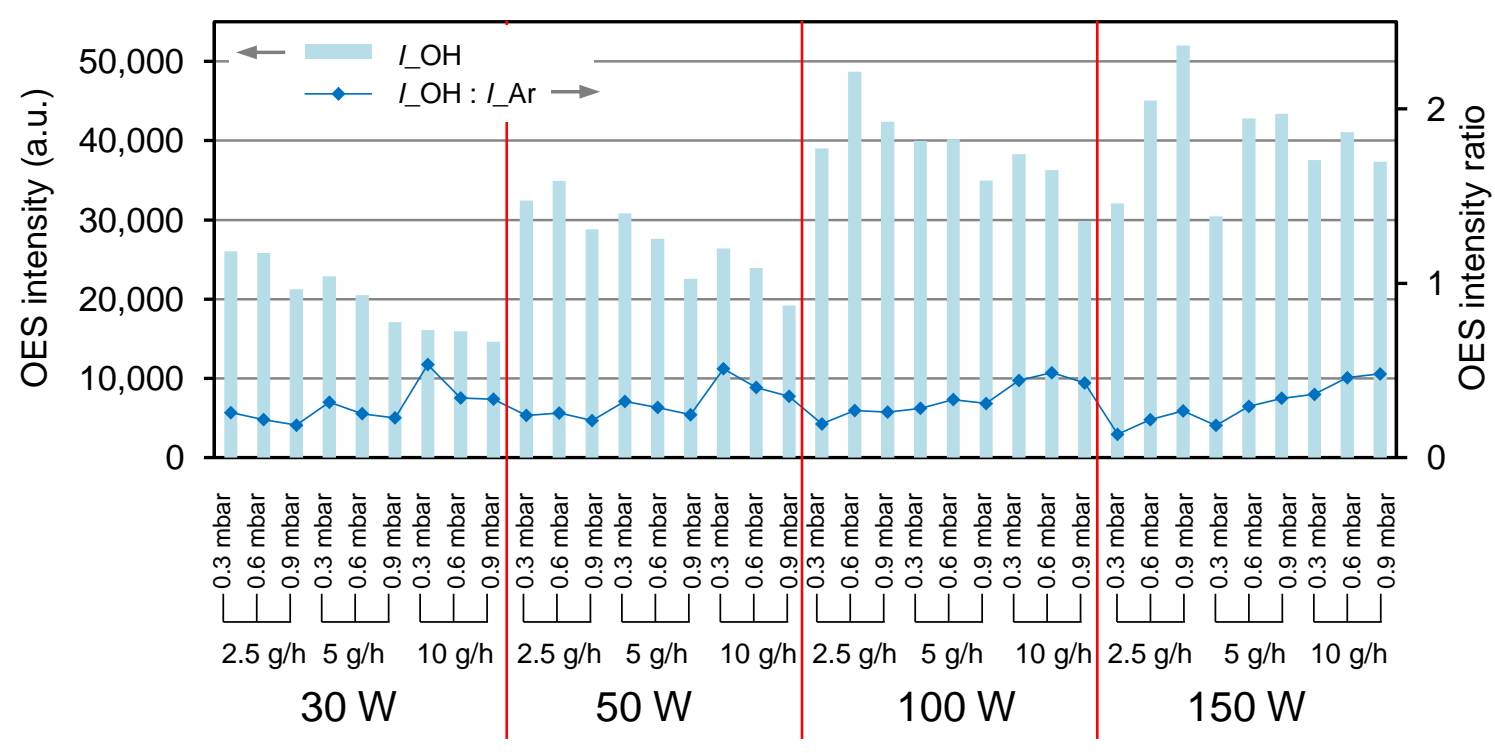

Figure 6 
Click here to download Figure: Figure7n_revised.pptx
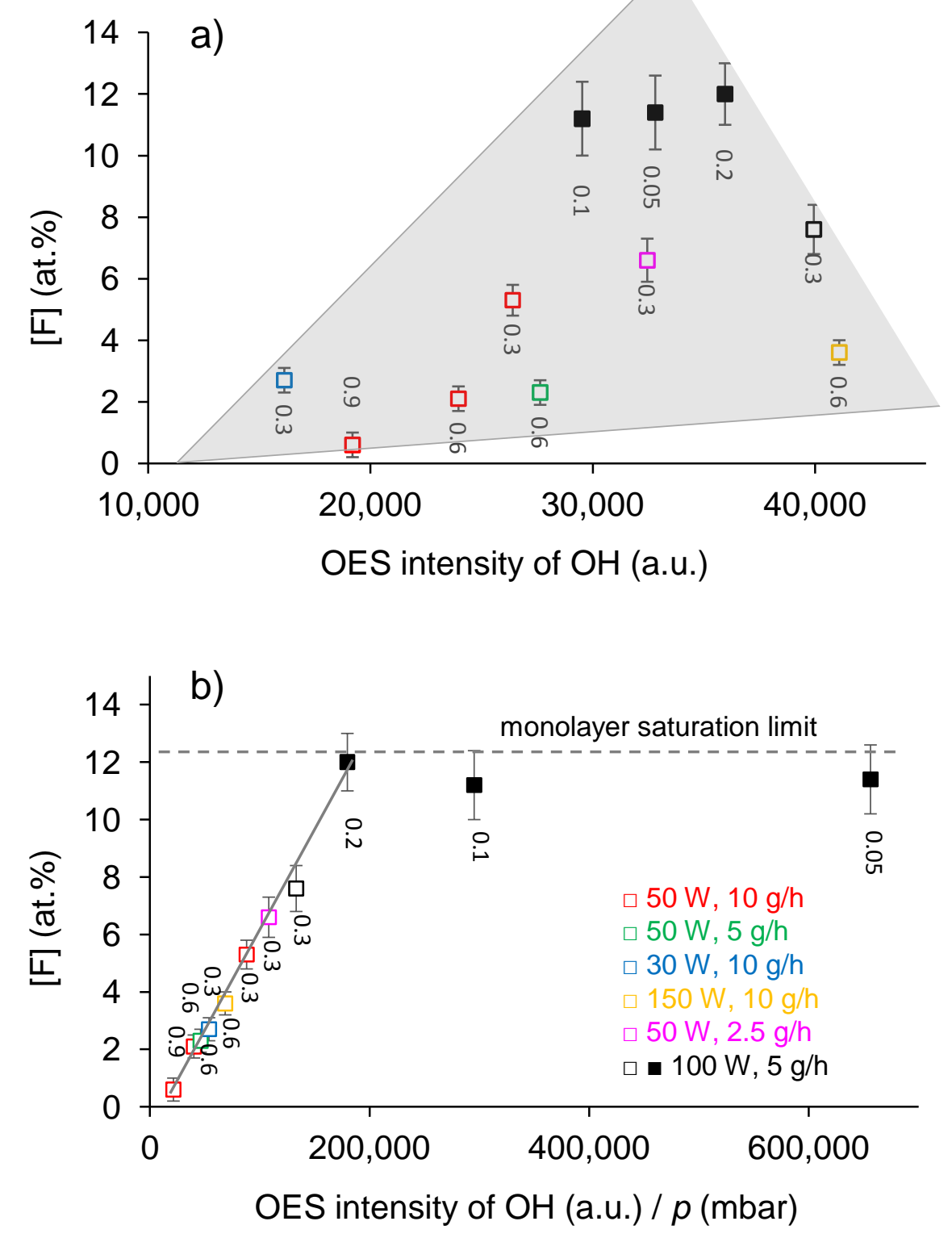

\section{Figure 7}

